\title{
LA NAVEGACIÓN PRE-ASTRONÓMICA EN LA ANTIGÜEDAD: UTILIZACIÓN DE PÁJAROS EN LA ORIENTACIÓN NÁUTICA
}

\author{
J. M. LUZÓN NOGUÉ \\ L. M. COÍN CUENCA \\ Universidad de Cádiz
}

\begin{abstract}
El presente estudio reúne los datos referentes a la utilización de pájaros (preferentemente cuervos y palomas) como auxiliares de los navegantes, para buscar la costa desde alta mar. Su utilización está documentada desde el tercer milenio a. C. En Oriente y durante el segundo milenio a. C. en el Egeo. De las posibilidades y limitaciones de esta forma de navegar depende en gran medida la interrelación cultural en el Mediterráneo. Un experimento prácticado realizado en 1986 permite comprobar la eficacia de la suelta de aves para orientar a los navegantes que se pierden en alta mar. La orientación astronómica supone la posibilidad de grandes rutas de ida y vuelta que van a cambiar radicalmente el panorama cultural y económico del Mediterráneo a partir del primer milenio a. C.
\end{abstract}

This paper deals with the use of birds (ravens and doves) as attendants of the sailors to search the coast from the sea. This use is documented since the III milenium B. C. in the Aegean Sea. The posibilities and limitations of this art of sailing are very important to the cultural interrelationship in the Mediterranean. An experience performed in 1986 has allowed us to verify the efficiency of the birds to guide sailors in high sea. The astronomical orientation assumes the possilibity of big return routes, which are radically changing the cultural and economic panorama of the Mediterranean since the I millenium B. C.

Desde hace ya bastantes años la investigación histórica y arqueológica han coincidido en aportar numerosos testimonios acerca de los elementos culturales de origen egeo, que aparecen en determinados lugares del Mediterráneo occidental durante la Edad del Bronce.

Sin duda el más llamativo de los focos con identidad propia lo constituyen los etruscos, en quienes se pretende reconocer numerosos rasgos lingüísticos (GEORGIEV, 1962; PALLOTTINO, 1968), religiosos (PERUZZI, 1968, 119) (1) y de cultural material (PERUZZI, 1968, 137), que llevan a considerar en cierto modo un origen oriental. Esto ya se veía así en la Antigüedad (HERODOTO, I, 94; ESTRABÓN, V, 2, 4; HORACIO Od, III, 29, 1), pe-

(1) Este autor aporta numerosos testimonios de esta índole para la formación de las culturas itálicas, a la luz de recientes investigaciones filológicas, históricas y arqueológicas. ro, no obstante, se originó una larga polémica que ha durado con múltiples matices hasta nuestros días (BLOCH, 1973, 67; TORELLI, 1984, 26) (2). En algunas ocasiones se ha llegado a afirmar que Etruria es «como un fragmento de Babilonia en suelo itálico», y en otras se ha minimizado el componente oriental en la formación lenta de una amalgama étnica plena de infiltraciones de todo tipo (HEURGON, 1981, 19).

Si realmente los etruscos tienen algún germen de identidad cultural en la llegada de unos Tursha (gentes mencionadas por los egipcios entre los denominados «pueblos del mar»), a las costas occidentales

(2) La polémica se originó en la Antigüedad con Dionisio de Halicarnaso $(I, 25,30)$ quien, contra toda la historiografía griega precedente, dice haber recogido entre los etruscos la idea de que son autóctonos y se llaman a sí mismos resenna y no tirrenos. 
de la península italiana, en torno al tránsito entre el segundo y el primer milenios antes de la Era (3), nos encontraríamos ante un núcleo más o menos numeroso de gente que llega por mar en una típica «navegación de fortuna» (4). Pero en tal caso hemos de considerar que no se trataría de un fenómeno único, sino repetido en muchas otras ocasiones, como la investigación arqueológica está desvelando en los últimos años (5).

Quizá el ejemplo más claro y aceptado de un fenómeno similar, en el que hacia las mismas fechas se origina en el occidente mediterráneo un foco cultural con identidad propia, pero también en clara dependencia con las culturas del Egeo, lo constituya la llamada Cultura Nurágica en la isla de Cerdeña (GROSJEAN, 1966, 190; FERRI, 1965, 261; LILLIU, 1962, 67). En muchos casos, la investigación arqueológica reciente ha tenido oportunidad de comprobar la presencia en Etruria de exvotos y armas de bronce, claramente relacionables con los que se conocen en el ambiente sardo de comienzos del primer milenio. Destaca entre ellos el modelo en bronce de un barco cargado de animales, especie de «arca de Noé», que bien pudiera evocar una llegada de las mencionadas en las fuentes antiguas y en la tradición (RINGEL, 1986, n. ${ }^{\circ}$ 54; GÖTTLICHER, 1978, n. ${ }^{\circ}$ 385-389; LILLIU, 1966). También es de gran interés una barca similar, hallada en Populonia asociada a una espada de bronce del tipo Monte Sa Ida (BIANCO PERONI, 1970, 99, n. ${ }^{\circ} 270$ ) y otra aparecida hace algunos años en el santuario marino de Gravisca (TORELLI, 1970, 57, n. ${ }^{\circ} 38$, lámina XVIII-a).

El hallazgo de un barco de bronce de tipo nurágico, ocurrido a 'mediados del siglo pasado en la desembocadura del Tíber, invita también a pensar en la existencia allí de un santuario visitado por navegantes desde comienzos del primer milenio antes de la Era (COLONNA, 1981, 171, lám. IX-b). Las re-

(3) Algunos aspectos de la navegación en el Mediterráneo hasta fines del segundo milenio y los paralelismos culturales y arqueológicos derivados de ella en MÜLLER-KARPE, 1982, 1 ss.

(4) Aunque la versión de Herodoto I, 94 ha sido muy discutida.

(5) Las múltiples arribadas a Italia, Sicilia y las islas de Córcega y Cerdeña están datadas a lo largo de toda la segunda mitad del segundo milenio a. de C. por los hallazgos de cerámica, lingotes de cobre, etc. Pero la explicación que habitualmente se da de que se trata de relaciones comerciales y contactos de intercambio a la manera que se impone a partir del siglo VIII o poco antes, está lejos de ser demostrada. Una visión de conjunto en la que se acepta este planteamiento en AUBET, 1984, 157 ss. laciones por mar entre Cerdeña y las costas occidentales de Italia durante la Edad del Bronce, son algo que parece tan lógico como comprobado por los hallazgos arqueológicos. Y ello es posible, precisamente, gracias a este tipo de barcos, de cuyo origen oriental hablaremos más adelante.

En el caso de Cerdeña se admite, por algunos autores, que nos encontramos ante un pueblo - los Shardana de las fuentes egipcias (SANDARS, 1978, 106) - que llega a occidente en la segunda mitad del segundo milenio a. de C., después de intentar inútilmente penetrar en el Nilo en barcos como los que más tarde reconocemos entre los bronces de la Cultura Nurágica (WACHSMANN, 1981, 187). El tema de esta identificación con los shardana fue ampliamente estudiado por Grosjean, quien analizó principalmente el paralelismo existente entre las espadas, cascos, escudos y corazas de los «pueblos del mar» en el relieve de Medinet Habú y las estelas funerarias sardas (GROSJEAN, 1966, 190 ss.; 1962, 1 ss.; ARNAL, 1976, 141 ss.)

Pero además del paralelismo anterior, ciertos hallazgos esporádicos apuntan igualmente a la llegada de elementos del Egeo. Algunoś de ellos indican un origen chipriota, como la esfinge de Nule o los lingotes de cobre en forma de «piel de toro» en los que hay grabadas marcas de escritura, que no dejan lugar a dudas sobre su origen (LILLIU, 1980, 344, lám. XLV-b; SANDARS, 1978, 101; BOUZEK, 1985, 19).

Desde un punto de vista estilístico, los rasgos orientales de la Cultura Nurágica fueron someramente esbozados por A. M. Bisi, quien relacionó diveros elementos de la Edad del Bronce de Cerdeña con las culturas anatólicas del segundo milenio a. de C. Entre ellos, nuevamente aparecen los barcos con unos prototipos que la autora remonta a modelos de barro conocidos en Kültepe. Son barcos abiertos que se distinguen por un prótomo en forma de cabeza animal (BISI, 1978, 349). Estas pequeñas embarcaciones abiertas, con mástil desmontable y caracterizadas por una cabeza de pájaro o por un prótomo en forma de cabeza de felino, se conocen en Medinet Habu (NELSON, 1942, 40; WACHSMANN, 1981, 187 ss; 1982, 297 ss.) y pueden considerarse técnicamente idénticas en el Egeo y en el Mediterráneo oriental.

Pero no es nuestro propósito desarrollar en este lugar la identificación por el mascarón de proa ( $\alpha \kappa-$

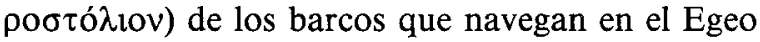
$y$ en el Mar Negro durante el segundo mileno antes de 
la Era. En todo caso, señalemos el paralelismo existente entre ellos y sus derivados occidentales de Cerdeña y Etruria (GÖTTLICHER, 1978) (6). Técnicamente se trata de pequeñas y rápidas embarcaciones, en las que se combina o alterna el esfuerzo de los remeros, con el impulso de una vela cuadra que se sostiene en un mástil abatible (GRAY, 1974, 100). Es decir, se trata de un barco abierto (la $\sigma \chi \varepsilon \delta i ́ n$ de Ulises) y mucho más primitivo que las grandes naves de carga y de guerra que se desarrollan aproximadamente desde el siglo VIII a. de C. (CASON, 1973, 53).

En barcos como estos hemos de imaginar la llegada de los pioneros que abandonan definitivamente el escenario del Mediterráneo oriental, hacia fines del segundo milenio antes de la Era. Son los relatos de la llegada de Eneas al Lacio, en los que se resume una serie de navegaciones escalonadas hacia Occidente, que la Arqueología está confirmado día a día, con el hallazgo de cerámica y objetos menores, datables principalmente en la fase final de las culturas Minoica y Heládica (HOLLOWAY, 1981; VAGNETTI, 1980, apéndice II; 1982, 19-20; AUBET, 1974, 157). En este contexto tienen cabida datos tan controvertidos como la fundación de Cádiz en el 1.100 a. de C. y muchos otros episodios, conocidos por las fuentes escritas, que la investigación moderna no ha sabido poner debidamente en orden (GARCÍA Y BELLIDO, 1948, 33 ss.; PEMÁN, 1931; 1954).

En un barco de estas características navega Eneas para auxiliar a la nueva Troya, llevando como distintivo de su proa dos leones. Le acompañan, entre otros, Másico, que corta el mar con la proa de su «tigre» y Astur que lo hace con un «caballo» (VIR-

(6) Sobre estas embarcaciones está en curso de preparación un trabajo monográfico de J. M. Luzón, en el que se abordan ampliamente las consecuencias históricas que aquí esbozamos.

(7) Hay que tener en cuenta que, aunque se ha discutido mucho la fundación de Cádiz en el 1.100, lo cierto es que en torno a esta fecha se asientan ex novo, en todo el valle del Guadalquivir, una serie de comunidades que van a desarrollar el Período Geométrico Andaluz. En esta etapa de ocupación, que cada día vamos detectando con mayor precisión a base de los sondeos estratigráficos, pudo haber un lugar que gozase del prestigio de haber sido el primer asentamiento. En la tradición se pudo mantener alguna leyenda originada en esta época tremendamente activa, pero no puede referirse en modo alguno a la llegada de los comerciantes tirios, que tiene lugar en el siglo VIII a. de C.

Un fenómeno similar y del mismo origen - aunque no podemos entrar en la compleja problemática que ello significa- lo encontramos, por ejemplo, en el País Valenciano (GONZÁLEZ PRATS, 1985, 153).
GILIO, Aen. $X, 155 ; 165$ y 181) (8), es decir, con un $i \pi \pi$ os. Virgilio alude en este episodio a un tipo de embarcación del que sólo pudo haber tenido un vago conocimiento literario. Lo mismo ocurre, como veremos más adelante, con determinadas referencias a los sistemas de navegación.

También en barcos como los que hemos visto tenemos que entender el desarrollo de la piratería tirrénica, que no es otra cosa que el traslado a Occidente de unas costumbres del mar y unas formas de vida, que han sido habituales en el Mediterráneo oriental, y quizá sea una de las causas que contribuyeron en mayor medida al colapso de la sociedad micénica (ORMEROD, 1978, 28 ss.; IENTILE, 1986).

Pero dejando a un lado los detalles del barco como tal, vamos a fijar nuestra atención en unos aspectos que generalmente se pasan por alto cuando tratamos de estudiar la navegación en la Antigüedad. Nos referimos a los animales que frecuentemente aparecen representados en los exvotos de bronce, tanto de Etruria como de Cerdeña a los que hemos aludido más arriba. En base a ellos hemos creido posible reconstruir algunos aspectos de lo que es la navegación de unos pioneros, que buscan tierras en las que asentarse, después de múltiples vicisitudes y singladuras errantes por unos mares que apenas conocen (9).

Lo primero que necesitan quienes marchan a nuevos lugares, bien porque emigran o porque pretenden explorar nuevas tierras, es llevar a bordo animales, semillas y aperos de labranza, con los que habrán de pasar el invierno, sembrar y recoger cosechas. Así nos describe Estrabón, siguiendo a Poseidonios, los preparativos de Eudoxos en su expedición por las costas africanas (ESTRABÓN, II, 3, 4) (10), y del mismo modo podríamos entender los numerosos animales que aparecen en un barco de la tumba del Duce, en Vetulonia, conservado en el Museo de Florencia (BIANCHI BANDINELLI GIULIANO, 1974, fig. 70; GÖTTLICHER, 1978,

(8) Aenia puppis / prima tenet, rostro Phrygios subiunta leones... / Massicus aerata princeps secat aequora tigri... / Astyr equo fidens et versicoloribus armis.

(9) La más detallada versión literaria de una de estas navegaciones, llenas de contingencias, la tenemos en la Odisea. Alvar (1981, 245 ss.) supone la orientación astronómica de los navegantes en fechas muy antiguas, a partir de conjeturas basadas en los constructores de Stonhenge. A nuestro juicio el argumento no es válido, ya que allí se trata de observaciones solares que no son útiles a la navegación. La astronomía estelar es muy posterior.

(10) Similares preparativos en Odisea, IX, 150-151 y 168-169. 


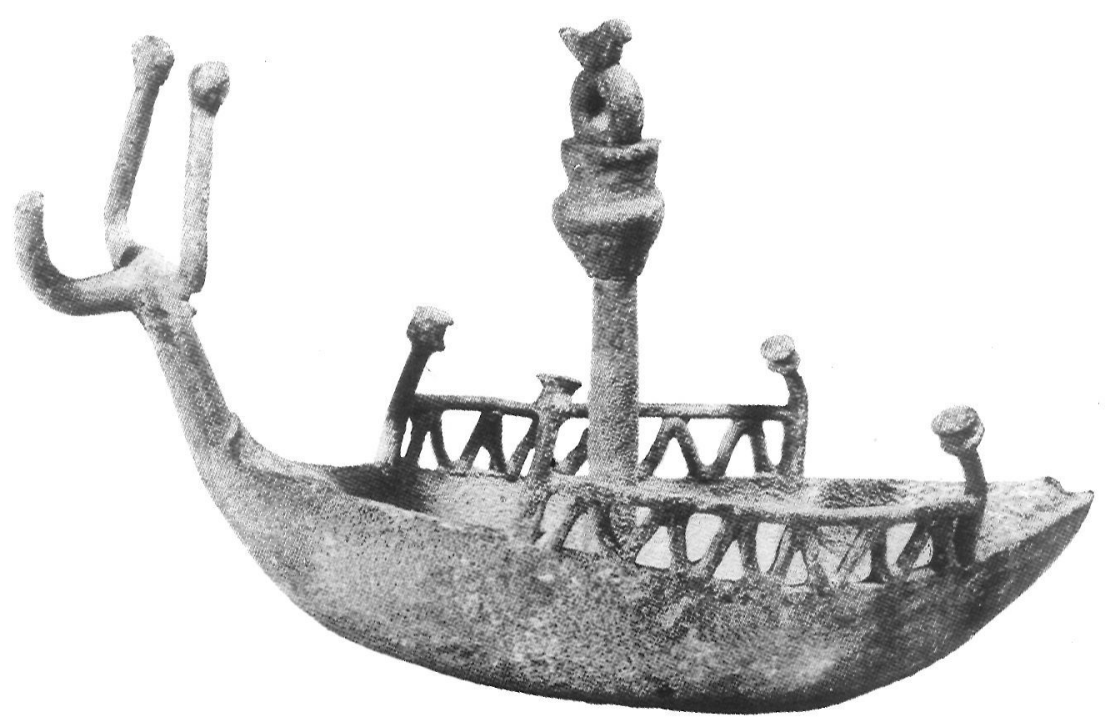

Fig. 1.-Barco con paloma posada en el mástil.

N. ${ }^{\circ} 428 ;$ RINGEL, 1986, n. $\left.{ }^{\circ} 54\right)$, y otro encontrado en Meana (Nuoro), que se guarda en el Museo de Cagliari (LILLIU, 1966, 327).

La presencia de algunos animales domésticos a bordo de una de estas pequeñas embarcaciones votivas resulta hasta cierto punto comprensible. Pero lo más frecuente es encontrar en ellas una o varias palomas, en el mástil o en la borda (Fig. 1), así como algunos macacos (Fig. 2), que hacen inconfundibles señas de divisar algo a lo lejos, bien apuntando en una dirección con el dedo índice, o bien llevándose la mano a los ojos en un gesto de escudriñar el horizonte (RINGEL, 1986, n. ${ }^{\circ}$ 54).

Los monos como auxiliares del navegante son algo conocido, pero de utilidad poco clara, debido a la parquedad de nuestra información. Sabemos, por ejemplo, que en Egipto son utilizados por los marineros, donde suele decirse, sin contar con fuentes fidedignas para tal afirmación, que eran amaestrados para subir a las partes difíciles del barco (BRUNNER-TRAUT, 1975; CASON, 1973, fig. 18; BARNETT, 1958, 223). En otros casos, nos ha llegado la representación conjunta de monos y pájaros, subidos a la antena de una nave de vela cuadrada, en un conocido vaso laconio del siglo VI a. de C., encontrado en Vulci, que se guarda en la Biblioteca Nacional de París (BOARDMAN, 1985, 43, lám. 203).

En todo caso, los barcos votivos de Cerdeña nos confirman que también son empleados por los marineros de la Edad del Bronce en el Mediterráneo occidental. Y quizá en barcos de fecha tan antigua pudieron llegar monos como los que luego describen en la isla de Pitecoussai (Ischia) los autores clásicos (OVIDIO, Met. XIV, 90).

Otro documento arqueológico de gran interés para determinados aspectos de la navegación es el tesoro de Egina, que se guarda en el Museo Británico, y del que haremos un análisis más detallado en otro lugar. En los pendientes aparece un barco sobre el que se sientan dos monos dándose la espalda.

Pero sin duda la parte más documentada, tanto en fuentes como en testimonios arqueológicos, es la relativa a los pájaros. Llevarlos a bordo puede ser el procedimiento más primitivo de seguridad que se conoce en la mar.

La versión más antigua de la utilización de pájaros para orientar al navegante perdido en alta mar la encontramos en el poema de Gilgamesh (CALMEYER, 1971; KEEL, 1977, 80 ss.; BARTRA, 1972, 118 ss.). La epopeya se refiere al diluvio, del que Utnapishtim se salva construyendo una nave con las maderas de su propia casa, siguiendo el consejo de los dioses. A nuestros días ha llegado en las versiones de los textos acadios encontrados en la biblioteca de Asurbanipal en Nínive, los fragmentos hititas de los archivos de Bogazköy y la versión babilónica antigua, que conocemos de manera fragmentaria. En todos los casos se trata de poemas que toman forma en el segundo milenio antes de la 


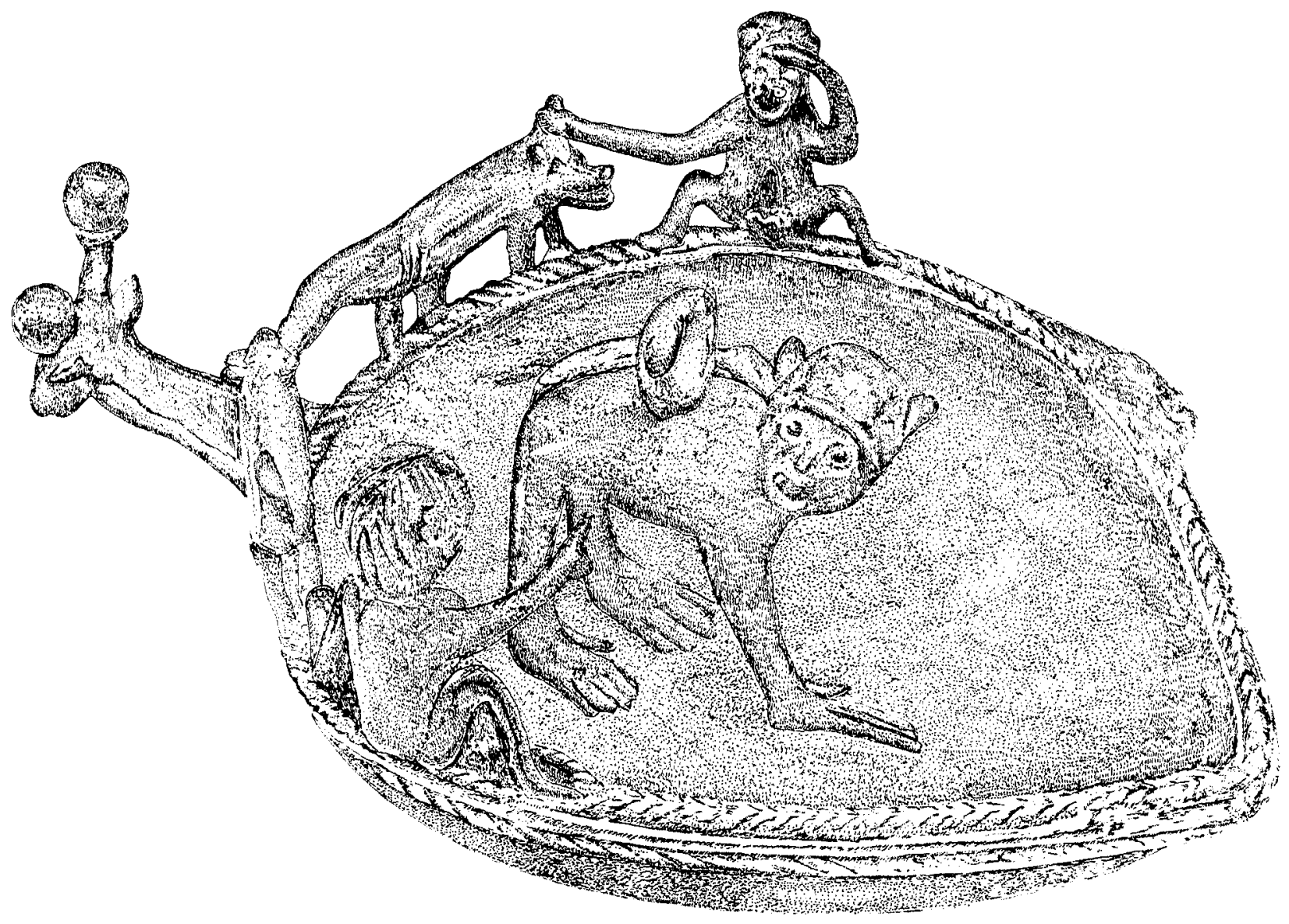

Fig. 2.-Barco votivo de Cerdeña.

Era, y que tienen su raíz en versiones no conocidas, pero de mayor antigüedad.

En la parte que nos interesa de esta epopeya, Utnapishtim es advertido para que se salve construyendo una nave: «Reúne en la nave la semilla de toda cosa viviente. / Que las dimensiones de la nave que has de construir / queden bien establecidas: / su longitud ha de ser igual que su anchura» (BARTRA, 1972, 121). A continuación hace subir a bordo animales y artesanos de todas clases, y comienza el pavoroso temporal que lo aparta de cualquier tierra visible. Finalmente, cuenta: «Al séptimo día, / la tempestad comenzó a ceder, / como un ejército en la batalla, / El mar se calmó, la tormenta amainó, / la inundación cesó. / Observé el tiempo: reinaba la calma / y la humanidad se había cambiado en barro / El paisaje aparecía liso como un techo».

En esta versión poética de cómo un navegante perdido busca la costa encomendándose a la voluntad divina, se cuenta que la nave queda encallada en la cima del monte Nisir, que yace aún bajo las aguas. Desde allí comienza Utnapishtim a buscar tierra: «Cuando llegó el sexto día, / solté una paloma. / La paloma emprendió el vuelo, pero regresó: / no había encontrado donde posarse. / Entonces solté una golondrina. / La golondrina emprendió el vuelo, pero regresó: / no había encontrado lugar donde posarse. / Entonces solté un cuervo. / El cuervo emprendió el vuelo, vio la mengua de las aguas, / corrió, resbaló, croó y no regresó. / Entonces hice que todo saliera hacia los cuatro vientos, / ofrecí un sacrificio, en la cumbre de la montaña...» (BARTRA, 1972, 122; KEEL, 1977, 84; FREEDMAN, 1973). Como vemos, el improvisado navegante busca tierra más allá de lo que sus ojos alcanzan a ver, soltando pájaros diversos y observando su comportamiento. Cuando, finalmente, uno de ellos no regresa, muestra su júbilo liberando las restantes aves que lleva a bordo y ofreciendo un sacrificio. El tema puede estar representado en un sello cilíndrico de la época de Mesilim, fechado 


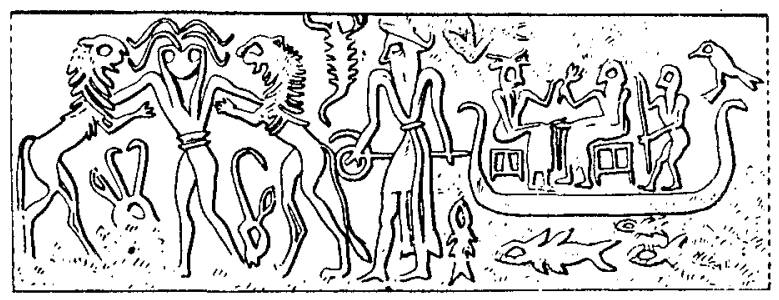

Fig. 3.-Desarrollo de un sello cilíndrico de Fara (según Frankfort).

hacia el 2.600 a. de C. procedente de Fara (Fig. 3) (KEEL, 1977, 83, f. 29; FRANKFORT, 67, lám. XI-m).

Tanto en el pasaje de Utnapishtim, como en los que vamos a resumir a continuación, se describe una extraña embarcación, aparentemente cuadrada, que será durante siglos una constante en narraciones similares. A veces se habla de una caja que flota en las aguas (11), pero no de un barco propiamente dicho. Por esta razón, cabe interpretar como representaciones relacionadas con el mismo episodio, unas embarcaciones cuadradas encontradas en el Karum de Kültepe, que se fechan en el siglo XIX a. de C. y que tienen en la proa una cabeza de carnero. En una de ellas hay un personaje masculino remando y en la otra se ve una figura que está haciendo ademán de subir. Como detalle significativo, en la borda de esta segunda hay posado un pájaro (Fig. 4) que bien pudiera ser la paloma que tan importante papel juega al final de la historia (BITTEL, 1976, 80, fig. 55, 91, fig. 71; BARNETT, 1958, 230; KEEL, 1977, 85, fig. 29).

En las anteriores cajas flotantes tendríamos, uno de los hitos cronológicos en los que apoyar la evolución histórica de la epopeya. Al mismo tiempo, es de gran interés comprobar el papel que juegan los colonos asirios de asentamientos como el de Kanish-Kültepe (ORTMANN, 1976-80), en la penetración hacia Anatolia de elementos culturales, que encontramos desde fecha muy antigua entre los hititas y los griegos, pero que tienen un claro origen mesopotámico.

Otra versión del diluvio, en la que se hace mención expresa de un barco nada convencional, y detallada descripción, de la utilización de pájaros para la localización de tierra, la tenemos en el conocido texto de Noé, adaptación semita de una historia muy difundida en Oriente. Según el relato bíblico, Noé, al no ver tierra desde el Arca, suelta primero

(11) En el mito de Deucalión, que comentamos más ade-

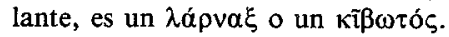

un cuervo y más tarde una paloma. Los hechos se describen en el Génesis de forma muy gráfica e inteligible: «...abrió Noé la ventana que había hecho en el arca, y para ver cuánto habían menguado las aguas soltó un cuervo, que volando iba y venía mientras se secaban las aguas sobre la tiera. Siete días después, para ver si se habían secado ya las aguas sobre la faz de la tierra, soltó una paloma, que como no hallase dónde posar el pie, se volvió a Noé, al arca, porque las aguas cubrían todavía la superficie de la tierra. Sacó él la mano, y cogiéndola la metió en el arca... pero todavía volvió a esperar otros siete días, y al cabo de ellos volvió a soltar otra vez la paloma, que ya no volvió más a él». (Génesis, 8, 6).

Del episodio anterior puede deducirse que, en el texto bíblico, hay recogida una antigua forma de navegación, que nada tiene que ver con la experiencia náutica en ríos y que muy verosímilmente habría que situar en Oriente, bien en el Mar Rojo o en el Mar Arábigo. Pero también es interesante señalar que en los dos primeros casos el cuervo y la paloma vuelan infructuosamente en todas direcciones, para regresar finalmente a la nave. Solamente concluye la navegación, cuando una paloma soltada por Noé se va definitivamente, es decir, cuando con su vuelo está indicando la dirección hacia la tierra que ha avistado.

Entre los griegos también se recoge en numerosos lugares la leyenda de un diluvio devastador del que solamente se salvan Deucalión y Pirra, gracias a un arca $(\lambda \alpha \dot{\rho} \nu \alpha \xi)$ en el que flotan durante nueve días. Nuestra fuente principal de información es Apolodoro (I, VII-3) (12), aunque el mito también es referido en otros lugares (13). El único autor grie-

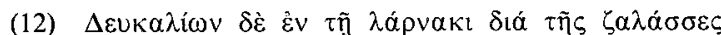

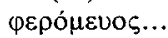

(13) La historia del Diluvio aparece de forma similar (LUCIANO Dea Syr., 12 ss.; OVIDIO, Met., I, 125-415). En Ovidio figura también un tal Carambus, que se salva (Met., VII, 353 , ss.) pero del que no se conocen más datos en los autores clásicos. Es posible que narraciones de este tipo, en lo que tie- 


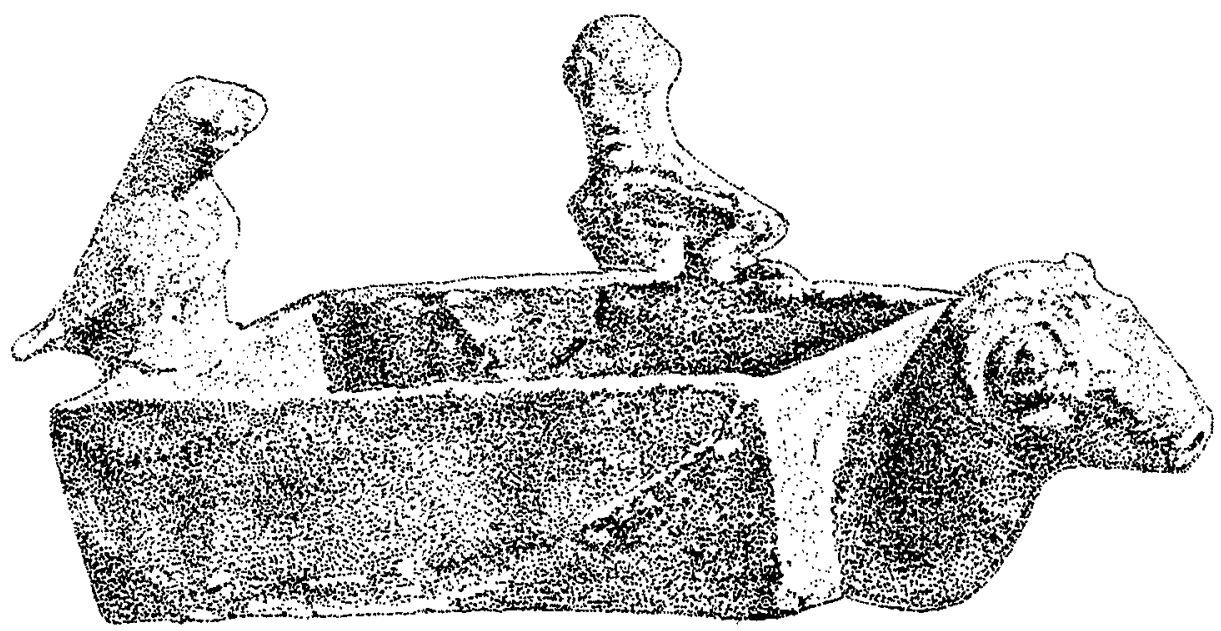

Fig. 4.-Barquita del Karum de Kültepe.

go que hace alusión a la suelta de aves desde el barco es Plutarco (Moralia 968) (14), quien dice que «Deucalión soltó una paloma $(\pi \varepsilon \rho ı \tau \varepsilon \rho \alpha ́)$ desde el arca, que mientras volvía era un signo cierto de que el temporal continuaba; pero tan pronto como se alejó volando, fue presagio de buen tiempo ( $\varepsilon u \delta i ́ \alpha) »$. Este episodio ha sido considerado como una contaminación semita, relacionada con el texto bíblico de Noé (CHERNISS y HELMBOLD, 1984, 376). Pero no debemos olvidar que esta historia, desde fechas muy antiguas, está configurada con todos los elementos esenciales en las culturas del Próximo Oriente. Lo que sí es significativo en el pasaje de Plutarco referido a la suelta de la paloma, es que éste no explica correctamente la utilidad que tiene para Deucalión. $Y$ es precisamente a partir de incomprensiones de este tipo como se comienzan a configurar los aspectos fabulosos y mágicos de una narración.

De manera mucho más explícita, encontramos la utilización de una paloma por parte de los argonautas en el episodio de las Simplégades. En la ver-

nen de saga, estén influenciados por las literaturas mesopotámicas, hurrita, hitita y cananea, pero ello no es óbice para entender que las técnicas de navegación son las mismas en el Mediterráneo (ADRADOS, 1987, 5 ss.)

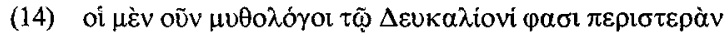

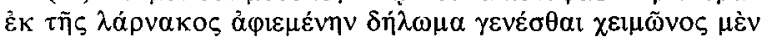

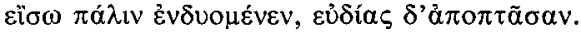

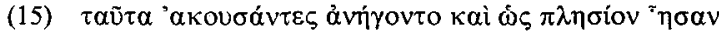

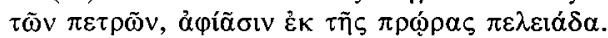

sión de Apolodoro $(I, 9,22)(15)$ los compañeros de Jasón sueltan una paloma desde la proa de su barco, para comprobar si iba a ser posible cruzar entre las rocas que chocan entre sí estrepitosamente. El embellecimiento poético de este episodio no impide reconocer el empleo de una paloma como orientación de los argonautas. Pero, además, podemos precisar que una historia así solamente puede haberse creado en la Edad del Bronce, y que su deformación es consecuencia del gran cambio que supone el tránsito de la navegación con pájaros a orientación mediante estrellas. Propercio (Eleg. II, $26,39)(16)$ conoce alguna versión cuando afirma que «una paloma suelta era la guía de la nave Argo en el mar desconocido».

Volviendo a la documentación que nos proporcionan los hallazgos arqueológicos, nos llama la atención un barco encontrado en Creta, que se guarda, en el Museo de Louvre (GÖTTLICHER, 1978, n. ${ }^{\circ}$ 143) (Fig. 5). Por su fabricación y elementos decorativos se considera obra chipriota del estilo «White Painted II» del Chipriota Medio I. En él aparecen ocho figuras en animado movimiento o quizá en estado de excitación o ansiedad, de las que una hace el gesto inconfundible de escudriñar el horizonte en la misma forma que encontramos uno de los monos de la barca nurágica de nuestra figura. Pero lo más llamativo de este modelo de terracota es que en la borda - como ocurre también en nu-

(16) cum ratis Argo Dux erat ignoto / missa columba maris. 


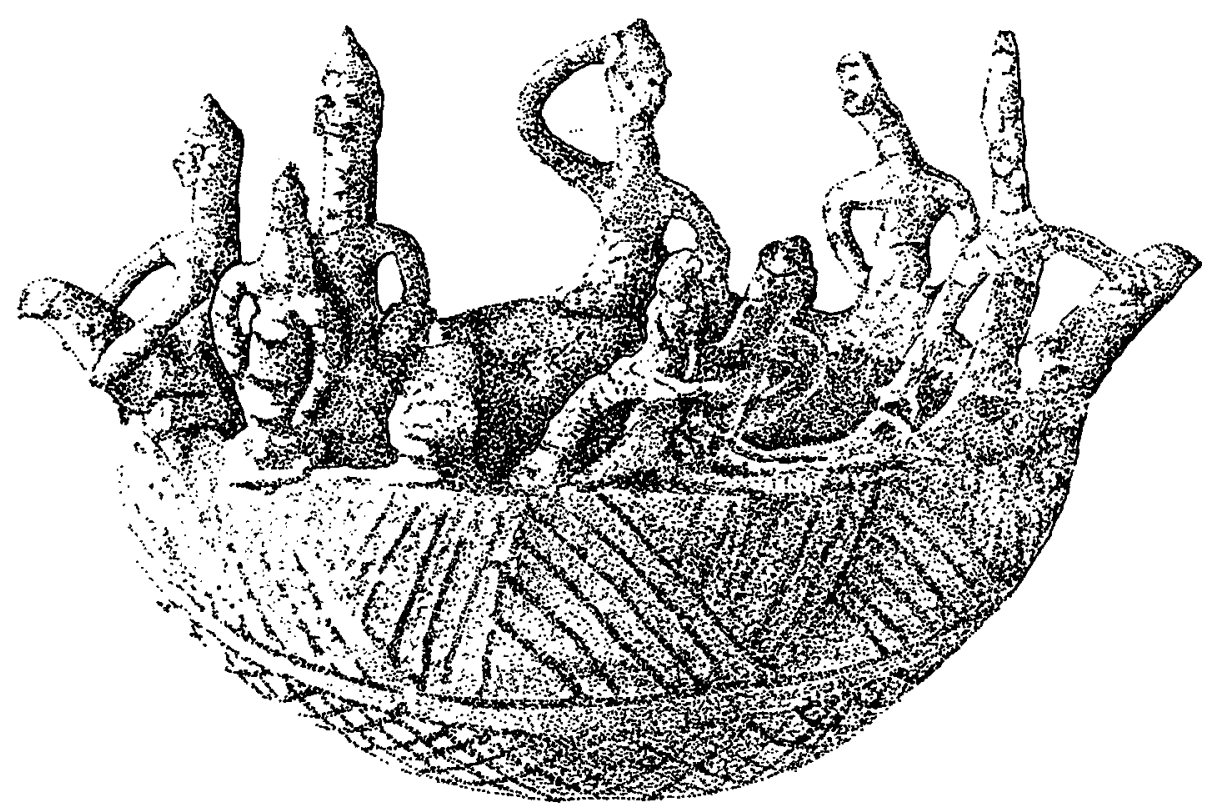

Fig. 5.-Barco votivo de terracota. Chipre.

merosos barcos de Cerdeña- se posan dos pájaros, en una clara alusión a este sistema de buscar tierra desde el mar. En tal caso, la figura que hace el gesto de mirar a lo lejos puede estar siguiendo el vuelo de una paloma, como luego veremos que hacen los compañeros de Eneas para llegar a las costas de Italia; quantum acie possent oculi seruare sequentum (VIRGILIO, Aen VI, 190-200).

Entre las numerosas historias que posiblemente tienen su origen en narraciones nacidas de la utilización de aves en el mar, cabe mencionar la contada por Filóstrato (Icon. II, 8) de cómo los calcídicos son conducidos hasta Cumas por las palomas (D'ARCY-THOMSON, 1936, 246).

En principio la utilización de pájaros por parte de los navegantes puede considerarse una fase preastronómica de la orientación náutica. Al menos eso es lo que parece deducirse de su desaparición en el Mediterráneo a comienzos del primer milenio antes de la Era. Aún así, la orientación a partir de la observación astronómica entraña el riesgo de que las nubes impidan una clara visión de los astros. Tengamos en cuenta que las condiciones de visibilidad del cielo no siempre son las más favorables para el navegante y, en ocasiones, se dan circunstancias tan adversas como las descritas en el viaje de San Pablo a Roma, precisamente por la pérdida de orientación: «En varios días no aparecieron el sol ni las estrellas, y continuando con fuerza la tempestad, perdimos al fin toda esperanza de salvación" (Act. $27,20)$.

Otras veces, como ocurría en la India, los pájaros eran utilizados a causa de las dificultades que tenían los navegantes para ver la Estrella Polar, dada la proximidad al Ecuador. Por tal motivo, se mantiene durante siglos esta forma de navegar y es recogida con detalle en un canon pali de Ceilán, unos cinco siglos antes de la Era, donde a los pájaros especializados en el menester de escudriñar la costa desde los barcos en alta mar se les denomina tiradassi (ANDRÉ-FILLIOZAT, 1980, 159).

De la misma fecha es la leyenda recogida por Hornell y otros autores (HORNELL, 1946, 143; BARNETT, 1958, 230; HUTCHINSON, 1962, 134; KEEL, 1977, 80; ALVAR, 1981, 643) en la Digha Nikaya, o primera parte del Sutta Pitaka hindú, en la que los mercaderes que tenían que navegar en mar abierto llevaban con ellos algunos pájaros (shoresighting birds), para localizar la tierra más próxima en caso de tener dudas acerca de la posición en que se encontraban. La narración más detallada de esta forma de navegar se encuentra en los Diálogos de Buda con Kevatta en Nalanda, escrito hacia el siglo $\mathrm{V}$ antes de la Era. En él dice Buda: «Hace mucho tiempo los mercaderes que iban al océano, a bordo de embarcaciones, llevaban con ellos pájaros buscadores de la costa (sakuna-). Cuando desde el barco no se divisaba la costa, soltaban el pá- 
jaro. Y entonces se ponía a volar hacia el este, hacia el sur y hacia el oeste y hacia el norte, y hacia los puntos intermedios, y se elevaba y daba vueltas. Si en el horizonte veía tierra se iba inmediatamente en esa dirección, pero si no la veía volvía de nuevo al barco» (KEEL, 1977, 80). Los pájaros que se utilizaban eran cuervos $k a k a-)$ y aparecen mencionados en otros textos contemporánes como los orientadores de los navegantes (disakaka-) (KEEL, 1977, 81).

En fechas más recientes, cuando los romanos describen las técnicas de navegación en Taprobane (Ceilán), insisten en la curiosidad de que navegan sin situarse mediante las estrellas. Así, Plinio el Viejo $(N H$, VI, 83) (17) explica que «los navegantes llevan pájaros a bordo, para soltarlos de vez en cuando y conocer por su vuelo la dirección de la tierra» (VERLINDEN, 1985, 21; McCRINDLE, 1901). En casi idénticos términos se expresa Solino $(53,7 ; 11$, 13) (18) y la misma curiosidad es recogida por Marciano Capella $(6,697)(19)$. El procedimiento empleado de forma habitual en el mar de la India es, como vemos, similar al que se describe en los distintos relatos del diluvio.

Conocida, pues, la utilización de pájaros en el Mar de la India, y utilizado por razones comprensibles más que en otros lugares, tiene sentido que existan leyendas de inundaciones en las que estos juegan un papel importante. Frazer cuenta la historia de una inundación recogida entre los kamar, tribu dravídica del distrito de Raipur, en la que pasado un tiempo Dios creó dos pájaros y los envió por todo el mundo. Los pájaros descubrieron un tronco con dos supervivientes, que fueron los progenitores de toda la especie humana (FRAZER, 1981, 107). Pero este autor, sin considerar que el empleo de aves por los navegantes puede haber dado origen a relatos como el mencionado, añade que la historia trae al recuerdo el episodio bíblico de Noé, por el incidente de las aves, y que éste pudo haber llegado a través de algún misionero.

(17) Siderum in nauigando nulla observatio; septentrio non cernitur. Volucres secum uehunt emittentes saepius meatumque earum terram petentium comitantur.

(18) Obsevatione itaque navigandi nulla suppetente, ut ad destinatum pergentes locum capiant, uehunt alites, quarum meatus terram petentium magistros habent cursus regendi... El mismo en 11-13.

(19) in nauigando nullum sidus obseruant, auium, quas uehunt, uolatus sequintur.
Precisamente se atribuye a la trasmisión de la leyenda del diluvio, una de las versiones más recientes con la representación del tema, en algunas monedas de Apamea-Kibotos, fundada por Seleuco Nikator, que corresponden a acuñaciones de Septimio Severo y Filipo el Árabe. En ellas se ven, dentro de una caja (кißßotóc), que flota sobre las olas, las figuras de un hombre y una mujer. Sobre la improvisada embarcación aparece posado un pájaro, mientras delante va volando otro que sujeta una rama entre las patas. La leyenda $\mathrm{N} \Omega$ ayuda a identificar a los personajes como Noé y Sambethe (TÜMPEL, 1905, 272, XVI).

Naturalmente, en el Mediterráneo, y sobre todo cuando ya se conocen a fondo sus vientos y sus corrientes, este método primitivo de orientación es mucho menos necesario que en los mares anteriores. Pero existen suficientes indicios de que los pioneros de la exploración hacia Occidente se guiaron por el vuelo de pájaros, para localizar las islas o la tierra firme, en mares a los que navegaban por vez primera. Así interpretamos los pájaros (quizá palomas) que aparecen en los barcos de bronce de la Cultura Nurágica que hemos descrito más arriba (Fig. 1). Y quizá en este contexto se pueda interpretar un relato virgiliano en el que las palomas sirven de indicio a Eneas y sus compañeros para concluir una larga y fatigosa singladura: «Tras pronunciar estas palabras, descendieron dos palomas volando desde el cielo, ante sus mismos ojos, y se posaron sobre el césped. Entonces el héroe máximo (Eneas) reconoce las aves de su madre (Venus) y alegre les implora: «jOh! sed guías, si es que hay algún camino, y dirigid el vuelo por los aires... y tú, divina madre, no me abandones en la incertidumbre... Habiendo hablado así, se paró observando los signos que le dan y el rumbo que toman. Ellas, comiendo, se alejan volando tanto como aquellos que las siguen son capaces de alcanzar con la vista» (VIRGILIO, Aen., VI, 190-200) (20). Aunque bien es ver-

(20) uix ea fatus erat, geminae cum forte columbae ipsa sub ora uiri caelo uenere uolantes, et uiridi sedere solo. tum maximus heros maternas agnouit auis laetusque precatur: «este duces, o. si qua uia est, cursumque per auras dirigite...

tuque, o, dubiis ne defice rebus, diua parens». sic effatus uestigia pressit obseruans qua signa ferant, quo tendere pergant. pascentes illae tantum prodire volando quantum acie possent oculi servare sequentum. 


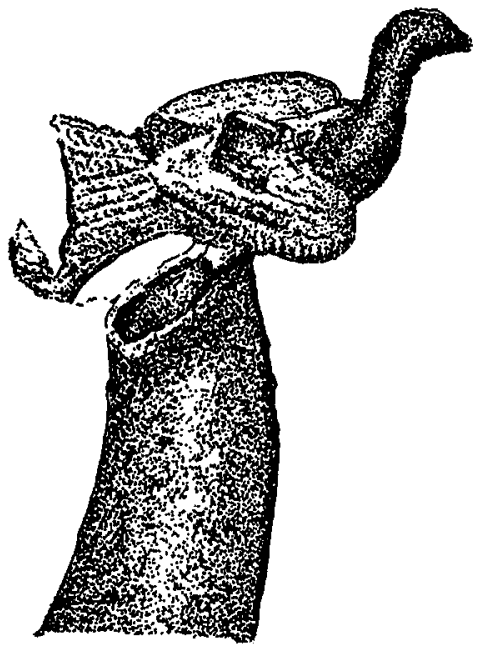

Fig. 6.-Paloma posada en un cuerno de Costix (Mallorca).

dad que Virgilio, inspirado en fuentes antiguas, insiste más en el carácter divino del augurio que en un procedimiento de orientación náutica que él mismo no comprende.

Para comprender el sentido religioso que la paloma y el mascarón de proa tienen para el navegante, hay un excelente ejemplo en la paloma que se posa en el cuerno de bronce de un toro de la cultura baleárica (FERNÁNDEZ MIRANDA, 1978, 270, lám. XXXV) (Fig. 6). Estos toros de bronce, dedicados en los santuarios, no son otra cosa que los

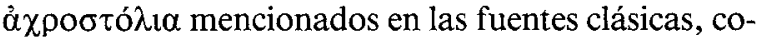
mo tendremos ocasión de tratar con detalle en otro lugar.

A comienzos del primer milenio antes de la Era se produce un importante cambio en la navegación, cuando se extiende para la orientación del piloto la observación nocturna de las estrellas. Con este cam-

En este caso nos encontramos con un texto en el que la forma poética y el sentido mágico religioso que se pretende dar al augurio, deforman unos datos que, en su esquema más simple, tiene aspecto de ser la orientación del Eneas navegante. En la narración virgilana se intercalan algunos elementos que no tendrían sentido, como es la aparición misma de las palomas enviadas por Venus desde el cielo. Igualmente anómalo resulta el hecho de que Eneas siga el vuelo de unas palomas que se paran de trecho en trecho a comer (pascentes). En una interpretación lógica de ese episodio, tendríamos que admitir la conexión misma que los marineros perdidos hacen entre el vuelo de los pájaros, de los que depende su suerte, y la protección de la divinidad a la que se encomiendan en el viaje. Datos como estos son tomados por Virgilio, con toda probabilidad en la Argonautica de Apolonio de Rodas, por quien se siente fascinado y profundamente influido en diversos episodios de la Eneida (LEVIN, 1971, 1). bio se dispone por vez primera del instrumento necesario para realizar grandes singladuras, y regresar al punto de partida, sin otras dificultades que las derivadas del estado del mar. Los griegos atribuyen la innovación, como nos dice Jenofonte, a los navegantes fenicios (Oeconomic. VIII; BARNETT, 1958, 230). Pero lo cierto es que unos y otros van a poder realizar navegaciones de ida y vuelta, que superan con mucho el mero deambular de Ulises o los argonautas, y abre así el camino para el establecimiento de las relaciones estables, que se documentan a partir de este momento. No es, pues, de extrañar que Atenea, la diosa que orientó a los marineros en forma de ave ( $\alpha i \theta v i \alpha$ ) (DETIENNE, 1970, 138 ss.), siga siendo la compañera inseparable del piloto que navega toda la noche con la vista fija en las estrellas (Odisea, 3, 12) (21), para mantener el rumbo (îuvelv) pero esta vez en su condición de diosa de visión nocturna $(\gamma \lambda \alpha \cup \kappa \tilde{\omega} \pi \mathrm{\jmath})$ ). En otras palabras, la orientación de los navegantes mediante las estrellas, es el cambio trascendental que va a posibilitar la llegada de comerciantes a las costas occidentales del Mediterráneo, y establecer las rutas de ida y vuelta en las que está basado el establecimiento de colonias griegas y fenicias, a partir del siglo VIII o quizá muy poco antes. Las llegadas anteriores no podían tener otro carácter que el de navegaciones sin retorno, en las que no existió en absoluto esa idea mercantil de las rutas marítimas, en las que algunos quieren basar la presencia de cerámica micénica en Italia (AUBET, 1984, 157 ss.) o de lingotes de cobre en Cerdeña (LILLIU, 1980, 344; BOUZEK, 1985, 19).

La asociación de Atenea, diosa protectora del piloto en la navegación, no se limita a la lechuza, sino que está también vinculada a la corneja y al cuervo. Posiblemente se base en que de todos los pájaros que se pueden emplear en la navegación la corneja es el que ofrece mayores ventajas, debido a que vuela más alto, es más resistente y resulta más fácil de seguir con la vista a gran distancia. Por ello en el culto de Atenea en la ciudad de Korone, puerto de mar en Mesenia cercano a la desembocadura del río Promis, la diosa aparece sentada y con un córvido (кopóun) posado en su mano (PAUSA-

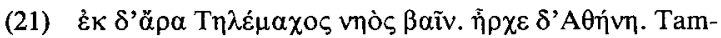
bién es interesante subrayar que la vinculación de Atenea con la corneja o el cuervo es anterior a la asociación con la lechuza, como se deduce de los episodios descritos por Ovidio (Met, 534541, 589-632). Sobre el tema RUIZ DE ELVIRA, 1982, 447. 
NIAS, IV, 34, 6) (22). En cuanto al cuervo es el ave oracular por excelencia, pero particularmente para los viajeros perdidos que demandan su ayuda (LUYSTER, 1965, 152). En este sentido podemos entender que en Orcómenos todavía se cuenten en época romana episodios como el que nos transmite Pausanias (IX, 38, 3) (23); con motivo de una peste que afectaba a los hombres y animales, los habitantes de la ciudad envían una delegación para que les indique lo que deben hacer. La sacerdotisa les dice que han de traer los huesos de Hesíodo desde la tierra de Naupactos a Orcómenos para frenar la calamidad que les azota. Los enviados le preguntan que de qué forma pueden conocer el lugar donde se encuentran los huesos. A ello les contesta la sacerdotisa que "un cuervo les indicará el lugar».

En un templo próximo a Sición recibían culto Atenea y Corone conjuntamente (PAUSANIAS, II, 11-12) (24). Una asociación similar a las anteriores es recogida por Ovidio (Met. II, 587-588) (25) en el episodio de la transformación de esta última en corneja. Neptuno quiere forzarla y ella pide ayuda, gritando a los dioses y a los hombres. Atenea, virgen como ella, corre en su auxilio y la convierte en ave para que pueda escapar de su perseguidor. Desde entonces, dice Ovidio, la tomó a su servicio.

La mayor antigüedad de las aves en la navegación que la observación nocturna de las estrellas puede estar reflejada en la desgracia en que cae la corneja en favor de la lechuza en los cultos y mitos de Atenea. El episodio más significativo lo tenemos quizá simbolizado en la lucha de las cornejas y las lechuzas descrito por Aristóteles ( $\mathrm{Ha}$. IX, 609a8) y Claudio Eliano (III, 9, V, 48) entre otros, y por el hecho de que las cornejas, según Claudio Eliano (III, 9; V , 48), no entraban en la Acrópolis de Atenas. La iconografía y los apelativos de Atenea como diosa del mar son elocuentes en el sentido que comentamos. La diosa es $\dot{\eta} \varphi \omega \sigma \varphi \rho^{\prime} \rho \varsigma$, «la lucífera», y también es aítula, pero tanto en una forma como en otra le conviene el apelativo de $\dot{\varepsilon} \kappa \beta \alpha \sigma i ́ \alpha$, diosa que conduce a los navegantes a buen puerto y diosa del «buen desembarco». En un vaso de Mikalessos

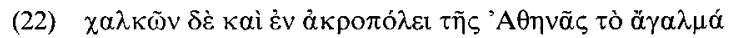

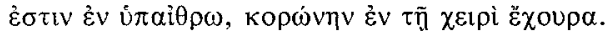

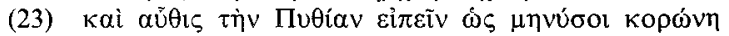

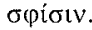

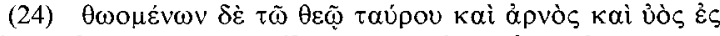

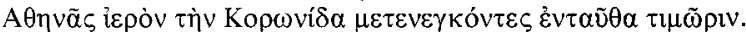

(25) mox acta per auras / euehor et data sum comes inculpata Mineruae.

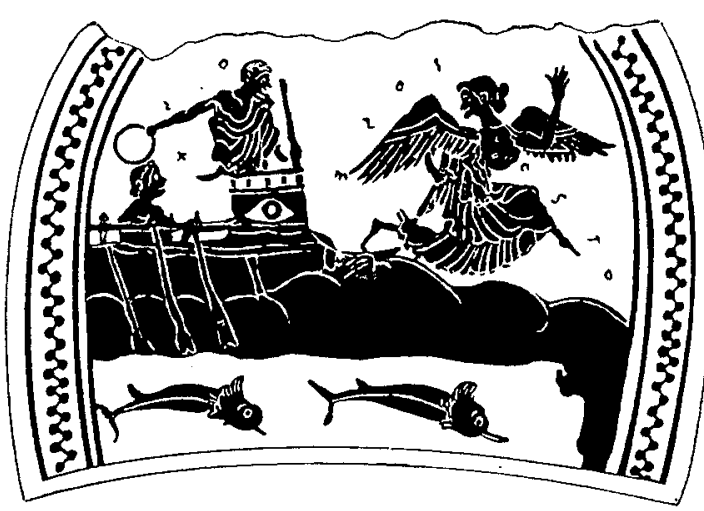

Fig. 7.-Vaso de Mikalesos (según Arias).

aparece una Atenea alada delante de la nave, guiándola al puerto en una iconografía que nos es conocida en otros ejemplos (ARIAS, 1920, 284) (Fig. 7). Posteriormente las estrellas que lleva en el peplos son sus elementos más característicos.

Como se puede comprobar, en el mundo griego y romano se reconocen relatos y costumbres de mucha antigüedad derivados, con el tiempo, de otras narraciones o ritos religiosos que tienen un sentido totalmente lógico y relacionados con la navegación en su forma más primitiva. Otro más de entre ellos sería la aparición de Ino a Ulises, también en forma de cuervo (Odisea, V, 333) aunque ello nos obligaría a analizar con más detenimiento el carácter oracular que tiene esta nave entre los griegos, debido quizá a la utilización que tuvo en tiempos muy antiguos por parte de los navegantes.

Conviene distinguir la utilización de pájaros para buscar la costa, del simple avistamiento de aves desde el mar, que con frecuencia sirve de indicador a los navegantes. Virgilio reserva esta señal de que la tierra está cerca, para la llegada de Eneas y sus compañeros a la desembocadura del Tíber: «En esto Eneas descubre desde el mar... en derredor y encima del río varias aves, acostumbradas a sus riberas y a sus aguas, (que) llenaban de dulces melodías el viento con sus gorgojeos y revoloteaban por el bosque. Allí manda Eneas a sus compañeros que tuerzan el rumbo, enderezando a tierra las proas, y se entre alegre por el umbroso río» (VIRGILIO, Aen., VII, 29, 36) (26). Los métodos del navegante pri-

(26) Aethera mulcebant cantu lucoque uolabant. / Flectere iter sociis terraeque adeuertere proras / Imperat et laetus fluuio succedit opaco.

La misma observación del vuelo de los pájaros sirve de indicio a los antiguos navegantes nórdicos para conocer la proximi- 
mitivo son recogidos y, en cierto modo desfigurados, para convertirse en signos inequívocos de la protección divina de Eneas.

Pero no es sólo, la orientación mediante aves que se sueltan desde el barco la que toma forma de presagio divino. También el camino fijado por las estrellas tiene su versión poética en la aparición milagrosa como guía y augurio profético: «... deslizándose del cielo en medio de las tinieblas, una brillante estrella. Después de resbalar por encima de nuestro palacio, la vimos esconderse clara en las selvas del monte Ida, señalándonos los caminos que debíamos de seguir» (VIRGILIO, Aen., II, 294-297) (27).

Un episodio similar, y cuyo origen puede estar ya muy apartado de la utilización práctica de los pájaros por parte de los navegantes, y más relacionado con las creencias religiosas que derivan de ella, es recogido por Silio Itálico, cuando Escipión parte de Sicilia y, desde alta mar, es guiado a tierra por el vuelo de unas águilas: «Entonces aparecieron ante la vista, volando por el claro cielo procedentes de la morada de los dioses, los pájaros que llevan los rayos de Júpiter, indicando a la flota el rumbo que debían seguir» (SILIO ITÁLICO, Punica, XVII, 5254) (28).

Es posible que, como tantas otras cosas procedentes del Egeo, en el Báltico se conociese desde fecha muy temprana la orientación en el mar mediante pájaros. Por ello los vikingos continúan utilizando, muchos siglos después, la suelta de cuervos, para seguir la dirección de su vuelo si no regresaban al barco (KRAPPE, 1936; HORNELL, 1946, 145; MARMS, 1953, 128). El caso más conocido lo tenemos en la saga del noruego Floki Vilgerdason, referida al descubrimiento de Islandia a mediados del siglo IX d. C. El episodio, narrado en la recensión de Sturla Thordarson al Landnámabók (Libro de los Asentamientos de Islandia) comienza con la consagración de tres cuervos a los dioses, a fin de que les sirvan de guías en el viaje que van a emprender. Después de navegar durante varios días hacia occi-

dad de la costa de Islandia, en sus navegaciones desde Hernar en Noruega hasta Hvarf en Groenlandia. La descripción de esta forma de orientar la ruta hacia occidente se describe en las versiones de Sturla Thordarso y Hauk Erlendsson del Landnámabók (JONES, 1985, 162).

(27) Stella ... multa cum luce concurrit ... signantemque vias.

(28) Tunc a sede deum parumque per aethera lapsae / armigerae lovis ante oculos coepere uolucres / aequoreas monstrare uias ac ducere classem. dente desde las islas Faroe, Floki suelta los tres cuervos a intervalos. El primero regresa al punto de partida, el segundo se eleva y ve desde la altura el horizonte vacio, por lo que regresa prudentemente al barco, y el tercero le guía con su vuelo a Islandia (JONES, 1985, 273; HORNELL, 1946, 145; ALVAR, 1981, 238 ss.) Nuevamente es el cuervo, como en otros ámbitos culturales, el ave que se considera particularmente capaz de conducir a los navegantes a tierra firme y ello hace que sea consagrado a Odín en la mitología nórdica.

Es interesante comprobar cómo los vikingos mantienen el primitivo procedimiento de buscar la costa utilizando aves, hasta fecha mucho más avanzada que en el Mediterráneo oriental. La explicación lógica es que la visión de las estrellas está mucho más limitada en el norte de Europa que en el Mediterráneo. Por ello el tránsito auténticamente innovador, que va a permitir navegaciones de ida y vuelta a lugares muy apartados, no se produce con la introducción de la navegación astronómica, que es en la que se basa toda la época de las colonizaciones griegas y fenicias, sino con la aparición de la brújula (HORNELL, 1946, 146).

Una orientación similar, mediante la observación del vuelo de las aves, era conocida, en el Atlántico norte, hasta época reciente, por los pescadores de las islas Shetland y de Islandia, cuando la visibilidad era escasa y la niebla les impedía ver la costa (SÖLVER-MARCUS, 1958, 32-33).

\section{LA SUELTA DE PÁJAROS DESDE EL BARCO-ESCUELA TARTESSOS}

Con los datos anteriores como punto de parti$\mathrm{da}$, consideramos suficientes los indicios de que el método oriental de la utilización de aves como auxiliar de los navegantes, había sido empleado en el Mediterráneo hasta algo entrado el primer milenio antes de la Era. Por ello, creímos conveniente realizar una experiencia práctica, para conocer los límites de un sistema de orientación tan elemental. Pretendíamos con ello matizar, e incluso desmentir, la idea extendida entre quienes se dedican al estudio del mundo antiguo, de que en casi todo el Mediterráneo existen alturas y montes visibles, que bastan al navegante experimentado, para cruzarlo en todas direcciones (SCHÜLE, 1968, 449 ss.). Esto es así por muchas razones, que los marineros profesionales conocen perfectamente. En parte, porque desde un barco de pequeñas dimensiones se tiene un punto 
de observación muy bajo, aunque se recurra a trepar a la cofa del mástil. Por ello las brumas, la evaporación, o incluso las nieblas bajas, limitan muy considerablemente la visibilidad del navegante. Además, el Mediterráneo, en los días de calor del verano, presenta la dificultad de una fuerte evaporación en los primeros metros por encima del agua, asi como la formación de neblinas y brumas que dificultan la visión mucho más que en tierra, aún en las mismas condiciones atmosféricas. De este modo, aunque el tiempo de navegación en la Antigüedad se limita, por razones de seguridad, a los meses del verano (29), tenemos que contar con que esa visión perfecta de los montes más alejados, ocurre durante muy pocos días. Nosotros mismos tuvimos ocasión de constatar las malas condiciones del mar durante el mes de julio, con temporales, nieblas bajas e incluso cielo totalmente cubierto durante algunos días. Gracias a ello la experiencia resultaba no solamente justificable, sino más variada de lo que en principio cabría suponer.

A las anteriores consideraciones hay que añadir que los pájaros se utilizan para buscar la tierra más próxima cuando el navegante está totalmente desorientado después de un temporal, pero en modo alguno para seguir con ellos una ruta previamente fijada. Es, por tanto, un procedimiento que en el Mediterráneo tiene cierto sentido en las navegaciones «de fortuna», a la búsqueda de nuevas tierras por aguas desconocidas. Es decir, que tiene una justificación en las arribadas que se dan en Occidente desde el Neolítico, y con muy pocas posibilidades de retorno. Lo mismo puede decirse de la navegación de Eneas y sus compañeros, o de la llegada de elementos orientales a la isla de Cerdeña o incluso a la Península Ibérica, detectados hace tiempo por la Arqueología en numerosos lugares (PERUZZI,

(29) Hesíodo (Trabajos y Días, 663-670) recomienda navegar sólo en julio y agosto. Otros autores limitan con menos rigidez el calendario de navegación, como Vegetius ( $R e$ Mil., IV, 49) da unas fechas de mayo a septiembre, para evitar los peligros del invierno, en que hay menos luz diurna y abundantes nubes: lux minima noxque prolixa, nubium densitas aeris obscuritas, uentorum imbri uel nauibus geminata saeuitia. En los Hechos de los Apóstoles, cuando se narra el accidentado viaje de San Pablo a Roma se atribuyen las malas condiciones del mar a que ya ha pasado el mes de septiembre y, por tanto, se ha entrado en el período en que la navegación es más peligrosa (Act., $27,9-10)$. Una precaución similar la encontramos en la protección que se invoca en época romana a la diosa Isis a la que dedican una especial liturgia el día 5 de marzo en que se abre la temporada de navegación (APULEYO, Met., XI, 5 y 16-17).
1980). Pero en absoluto se pueden explicar como resultado de rutas establecidas y fijas, a la manera de las que se empiezan a consolidar a partir de la época orientalizante (PICARD, 1982, 167 ss.)

Para la experiencia utilizamos el barco-escuela Tartessos de la Escuela Superior de Náutica de Cádiz (30) y una serie de aves diversas. Contábamos con dos milanos, un cuervo, dos grajillas, seis tórtolas y palomas comunes todas ellas anilladas por la Estación Biológica de Doñana (31). Además llevábamos once palomas mensajeras facilitadas por la Sociedad Colombófila de Puerto Real (Cádiz) (32). En la práctica, como detallaremos más adelante, sólo fueron útiles los vuelos de las palomas, debido a que se encontraban en libertad hasta un par de días antes de nuestra partida. No así las restantes aves, que llevaban excesivo tiempo en cautividad y no se encontraban en condiciones de volar a largas distancias.

Nuestro propósito inicial era el de hacer una ruta desde Cádiz hasta Ibiza (Fig. 8), adentrándonos en puntos apartados de alta mar, para tratar de comprobar a qué distancia podía ser efectivo este rudimentario procedimiento de buscar tierra. De igual modo nos interesaba conocer la utilidad de los pájaros en distancias cortas, pero con la visibilidad horizontal muy limitada por las brumas o nubes bajas.

Salimos de Cádiz el día 17 de julio de 1986 y regresamos el 30 del mismo mes, tras realizar las pruebas cuyo resultado extractamos a renglón seguido:

1) A las $6,08 \mathrm{~h}$. (GMT) del día 21, en posición $\mathrm{I}=36^{\circ} 16^{\prime} \mathrm{N} . \mathrm{L}=04^{\circ} 41^{\prime} \mathrm{W}$, cuando por la mañana ya había claridad suficiente como para seguir visualmente el vuelo de los pájaros, soltamos la primera de las palomas, a fin de tener una impresión acerca del comportamiento de vuelo en condiciones de visibilidad relativamente favorables, y después de la dureza de un temporal en contra, de levante, en el Estrecho de Gibraltar. Durante la noche habíamos tenido unos vientos de fuerza 8 de la escala de

(30) Agradecemos a la Dirección General de la Marina Mercante y a la Escuela Superior de la Marina Civil de Cádiz las facilidades para la utilización del barco escuela «Tartessos».

(31) Nuestro agradecimiento a la estación biológica de Doñana donde accedieron a anillar todas las aves empleadas en la experiencia, con los números de referencia utilizados en este centro de investigación.

(32) Agradecemos al Dr. Antonio Caro de la Universidad de Cádiz, la cesión de palomas comunes de su propiedad y a D. José Jiménez de Puerto Real, por las palomas mensajeras y sus valiosas orientaciones sobre el tema. 


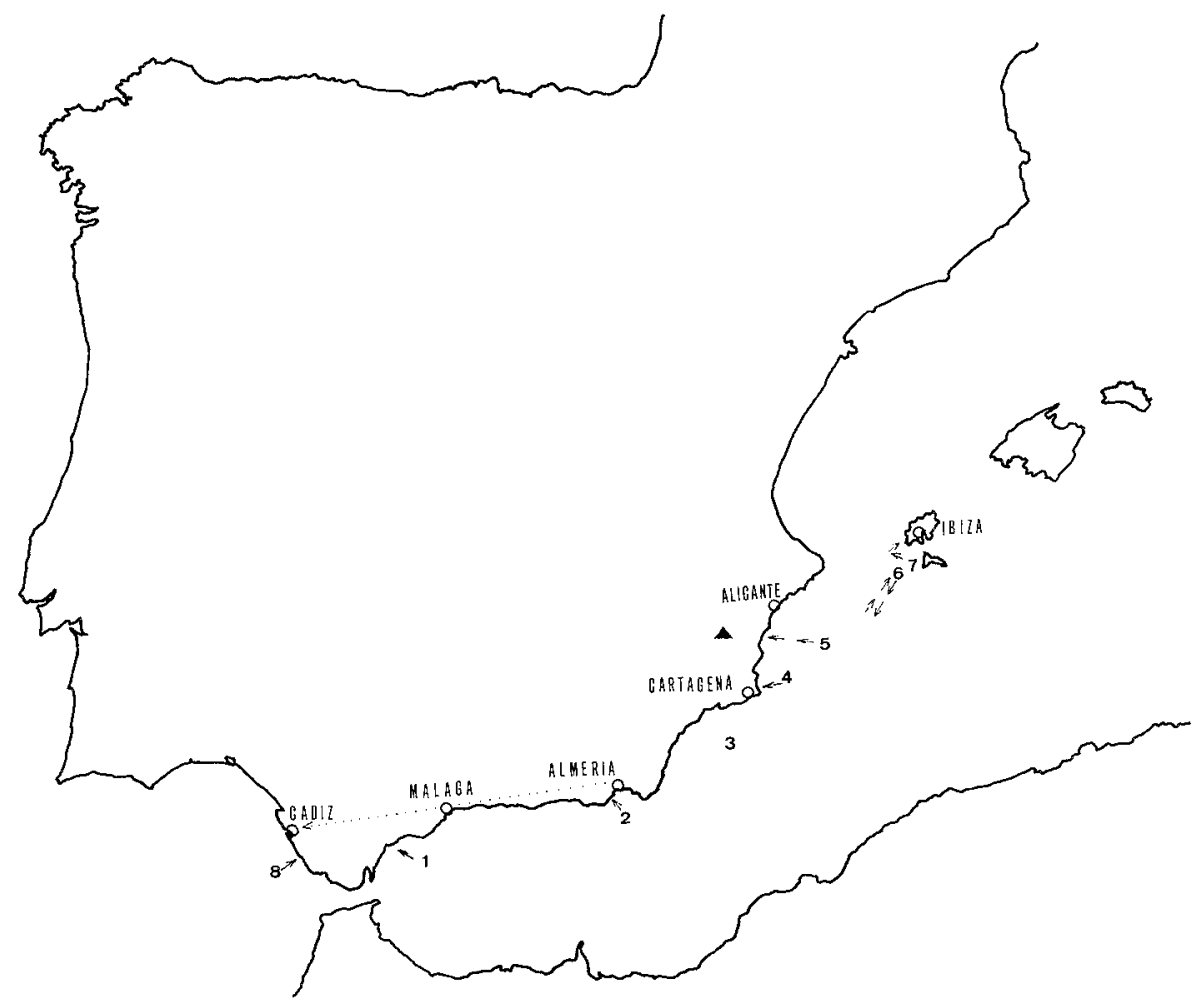

Fig. 8.--Mapa del itinerario con indicación de los lugares de suelta de palomas.

Beaufort ( 40 nudos $=74 \mathrm{Km} / \mathrm{h}$ ) y olas de 5 metros de altura también de levante. Esta circunstancia es particularmente resaltable, por el hecho de que en tales condiciones, los pájaros habían estado expuestos a los continuos rociones de las rompientes de las crestas de las olas, a pesar de tener las jaulas muy protegidas con lonas. Por ello nos parecía que podían haber perdido cierta capacidad de vuelo.

En el momento en que soltamos esta paloma las condiciones náutico-atmosféricas eran de una ligera bruma, con buenas condiciones de visibilidad en altura, que permitían ver claramente las cimas de las Sierras de Mijas, Marbella y Estepona. Navegábamos con rumbo E-NE a una velocidad de $6 \mathrm{nu}$ dos $(11 \mathrm{~km} / \mathrm{h})$. El viento era del $\mathrm{E}$ y tenía una velocidad de 9 nudos $(17 \mathrm{~km} / \mathrm{h})$.

La paloma fue soltada por la banda de babor, que era la del lado de tierra, y era previsible que dirigiese su vuelo hacia el $\mathrm{N}$, donde la distancia a costa era de 15 millas $(28 \mathrm{~km})$. Sin embargo, salió con dirección $\mathrm{W}$, donde también se veía tierra, aunque a una distancia de treinta millas $(55 \mathrm{~km})$. Hizo un vuelo muy recto y rápido en la misma dirección del vien- to y se perdió de vista sin cambiar el rumbo de vuelo (Fig. 8).

Tras esta primera vuelta comprendimos las posibles ventajas que tendría el hacerlas con el barco parado en las fases siguientes, así como la importancia que podría tener la fuerza y dirección del viento a la hora de valorar los rumbos escogidos por los pájaros.

2) A las 10,55 h. (GMT) del día 22 de julio hicimos la suelta de las once palomas mensajeras cedidas por la Sociedad Colombófila de Puerto Real. Esta operación se hizo con el barco parado, en posición $\mathrm{I}=36^{\circ} 47^{\prime} \mathrm{N}$ y $\mathrm{L}=02^{\circ} 30^{\prime} \mathrm{W}$, a una distancia de tres millas $(5,5 \mathrm{~km})$ de la costa en dirección $\mathrm{W}$, que era la línea recta de vuelo hacia el lugar de procedencia, y de dos millas $(3,7 \mathrm{~km})$ en dirección $\mathrm{NW}$, donde estaba la tierra más próxima, con los montes de Almería perfectamente visibles. Fueron soltadas en el espacio de nueve minutos, con muy cortos intervalos, y el comportamiento de ellas fue siempre el mismo: procedían a elevarse haciendo un semicírculo, e inmediatamente dirigían su vuelo hacia la costa por el lado en que ésta se encontraba a menor distancia. Una vez en tierra orientaron su 
vuelo hacia Puerto Real (Fig. 8). Las que llegaron lo hicieron todas ellas dentro del día.

Hay que tener en cuenta que las palomas mensajeras están especialmente entrenadas por sus cuidadores para vuelos largos. Para ello se las obliga a salir del palomar varias horas todos los días y se les prepara la ración de comida y agua al final de cada vuelo. De este modo están en condiciones físicas para someterlas a los vuelos de largos trayectos. El dato tiene interés, porque nos hace comprender que en la Antigüedad, si se quería disponer de aves destinadas a una misión de vuelo en las que el esfuerzo físico debería ser igualmente importante, lo lógico es pensar que se saliese al mar con ellas sometidas al entrenamiento descrito. Pero no creemos que la paloma mensajera sea la adecuada para buscar la tierra más próxima, ya que en alta mar puede orientarse hacia su palomar desde distancias muy largas, sin dirigir previamente el vuelo a la costa. Con todo, es posible su utilización con el fin más concreto de localizar un puerto o una isla determinada.

3) El día 23, a las 9,30 h. (GMT), en posición $\mathrm{I}=37^{\circ} 10^{\prime} \mathrm{N}$. $\mathrm{L}=00^{\circ} 50^{\prime} \mathrm{W}$., con una visibilidad escasa, debido a la bruma, y una distancia a tierra -que no era visible - de 15 millas $(28 \mathrm{~km})$, intentamos la suelta de algunas aves de distinto tipo. Se trataba de dos milanos y un cuervo cedidos por el Zoológico de Jerez.

En los tres casos las aves comenzaron remontar el vuelo, pero se agotaron al cabo de muy poco tiempo. Iniciaron el retorno al barco, pero no llegaron a posarse, por lo que tuvimos que rescatarlas del agua y volverlas a enjaular, para su posterior devolución. De todas formas consideramos de interés la constatación, una vez más, de que las condiciones físicas y el entrenamiento de los pájaros resultaba determinante para su utilización en las navegaciones antiguas. La seguridad de los navegantes depende, en casos como éste, de la capacidad de vuelo de las aves empleadas.

4) A las 13,00 h. (GMT) del mismo día 23 soltamos dos palomas comunes desde la posición $\mathrm{l}=37^{\circ} 40^{\prime} \mathrm{N} . \mathrm{L}=00^{\circ} 30^{\prime} \mathrm{W}$. Iban anilladas con los números 006556 y 006565 de la Estación Biológica de Doñana. Estábamos a 9 millas $(17 \mathrm{~km})$ de Cabo de Palos, que era el punto más cercano de la costa. La visibilidad era muy buena y el viento del $\mathrm{S}$ tenía una fuerza 4 de Beaufort (16 nudos $=30 \mathrm{~km} / \mathrm{h}$ ).

La primera de ellas trazó un semicírculo a baja altura, e inmediatamente puso rumbo hacia tierra, elevando paulatinamente el vuelo. Cuando ya se había perdido de vista esta primera paloma, soltamos la segunda, que tuvo un comportamiento idéntico (Fig. 8).

Con la suelta de estas dos palomas comprobamos que en condiciones de viento en contra no tenían dificultad en dirigir su vuelo hacia tierra. Igualmente vimos que las palomas estaban en buenas condiciones para realizar las fases sucesivas de la experiencia.

5) A las 18,05 (GMT) nos encontrábamos en posición $\mathrm{I}=37^{\circ} 55^{\prime} \mathrm{N}$. $\mathrm{L}=00^{\circ} 03^{\prime} \mathrm{E}$. La distancia más corta a costa era de 35 millas $(65 \mathrm{~km})$, en el Cabo de Santa Pola (Alicante) y la visibilidad desde barco era de 10 millas $(18,6 \mathrm{~km})$, aunque desde mayor altura podrían verse las crestas de la Sierra de Crevillente, que se encontraba a 45 millas $(84 \mathrm{~km})$ en línea recta. El viento era del $\mathrm{S}$ y tenía una fuerza 2 de Beaufort ( 5 nudos $=9 \mathrm{~km} / \mathrm{h}$ ).

En estas condiciones soltamos una paloma anillada con el número 006562 (Fig. 6). En un primer momento se dirigió hacia el SE en un vuelo bajo y recto. Se trataba de un rumbo que estaba ligeramente en contra del viento. A continuación cambió de dirección, describiendo un círculo hacia el $\mathrm{W}$, y fue elevándose progresivamente. A una cierta altura fijó definitivamente el rumbo de vuelo hacia el $\mathrm{NW}$, en línea recta a la Sierra de Crevillente $(836 \mathrm{~m}$ de altura) -que nosotros no veíamos desde el barco-, alejándose en esa dirección hasta perderse de vista (Fig. 8).

Con el vuelo de esta paloma comprobamos que era perfectamente posible fijar la posición de la tierra más próxima desde una distancia bastante considerable para una navegación rudimentaria. En este caso estábamos a 35 millas $(65 \mathrm{~km})$.

6) El día 24 a las $05,10 \mathrm{~h}$. (GMT) estábamos en posición $\mathrm{I}=38^{\circ} 4^{\prime} \mathrm{O}$. $\mathrm{L}=01^{\circ} 05^{\prime} \mathrm{E}$, que corresponde a una distancia de 15 millas $(28 \mathrm{~km})$ de las islas de Ibiza y Formentera, pero con mala visibilidad, que no llegaba apenas a las dos millas $(3,7 \mathrm{~km})$ y con un viento del S SE de fuerza 4 de Beaufort ( 15 nudos $=28 \mathrm{~km} / \mathrm{h}$ ). Durante la noche habíamos pasado un fuerte temporal de mar y viento de SE, con grandes balances del barco, que podían haber influido en las condiciones de vuelo de las palomas.

En estas circunstancias soltamos una paloma, con número de anilla 500657, por la amura de la banda de estribor, en la dirección de las islas, que no eran visibles desde el barco. Inicialmente se dirigió contra el viento, describiendo un semicírculo ha- 
cia el S y luego hacia el SW por donde se perdió de vista en un vuelo recto y alto. Es decir, la paloma se había orientado hacia alta mar y se había perdido por popa en dirección contraria a nuestro rumbo. Está claro que había escogido la dirección de vuelo menos adecuada para encontrar tierra, por lo que resultaba evidente que las nubes bajas o estratos le impedían la visibilidad de las islas desde la altura en que se situaba. Las islas, además, estaban más cubiertas de nubes que el resto del horizonte, por lo que tenía una cierta lógica que volase a la búsqueda de tierra en la dirección indicada.

Pasado algún tiempo, y visto el comportamiento de la paloma que habíamos soltado, la dimos por perdida y anotamos el resultado negativo de la experiencia. Sin embargo, a las 05,30 h. (GMT) la paloma apareció por la popa, siguiendo nuestro rumbo hasta posarse en el aparejo de barco. La llovizna la había mojado y el esfuerzo que había realizado hizo que no ofreciese resistencia para cogerla y enjaularla nuevamente (Fig. 5).

Ante la sorpresa que suponía para nosotros el regreso de una paloma que no había encontrado tierra, cuando ya no la esperábamos, volvimos a repetir la experiencia con una segunda a las $05,40 \mathrm{~h}$. (GMT). Iba anillada con el número 50064. Estábamos a una distancia de 11 millas $(20 \mathrm{~km})$ de las islas y con las mismas condiciones atmosféricas. El comportamiento de esta paloma fue muy similar al de la anterior, perdiéndose por la popa, en dirección a mar abierto. Regresó a los diez minutos de vuelo y se intentó posar en el tope del mástil, cosa que no lograba por los balances y por resbalar en la superficie de aluminio. Nuevamente intentó un segundo vuelo en dirección $\mathrm{N}$ sin llegar a ver las islas a causa de la niebla. Por este motivo volvió a aparecer nuevamente de regreso al barco. A los pocos minutos salió por tercera vez, pero en esta ocasión en dirección $\mathrm{W}$. Tambén en este último vuelo llegó a perderse de nuestra vista, pero tardó menos en regresar que las veces anteriores. Al tratar de enjaularla se fue hacia un velero de bandera inglesa que se cruzaba con nosotros a media milla aproximadamente.

Resulta de interés constatar el hecho de que las aves que no encuentran tierra donde posarse, ni la ven desde la altura de su vuelo, vuelven nuevamente al barco del que partieron. Para el navegante de la Antigüedad este hecho es un dato de tanta importancia como la indicación de tierra en una dirección determinada, aunque obviamente más desesperanzador si se encontraba perdido. Es decir, que el pájaro se aleja definitivamente tan sólo en el caso de que haya tierra en algún lugar al alcance de su vista. Comprobábamos con ello la veracidad de la situación descrita en los episodios de Utnapishtim, Noé y Deucalión.

7) A las 07,00 h. (GMT) nos encontrábamos ya a 4 millas $(7,4 \mathrm{~km})$ de distancia del Cabo Falcó (Ibiza) y demora verdadera S-SE de dicho punto, con una visibilidad de 2 millas $(3,7 \mathrm{~km})$, por lo que no se veía aún la tierra desde el barco.

En la anterior posición soltamos una paloma anillada con el número 5006551, que describió un vuelo en semicírculo y se dirigió recta hacia un barco contenedor que estaba a unas dos millas de distancia al W del nuestro. A mitad de vuelo, en aquella dirección, corrigió su rumbo y se fue directamente a tierra, que pudo avistar por algún hueco de mayor claridad entre las nubes. Se perdió de nuestra vista y no volvió más, con lo que estaba indicando la dirección correcta a la tierra más próxima, que nosotros teníamos perfectamente localizada en el radar de a bordo (Fig. 8).

A continuación soltamos una segunda paloma con el número de anilla 5006561 , que repitió un vuelo idéntico al anteriormente descrito. Con ello confirmábamos que en aquella dirección había tierra visible para las aves (Fig. 9).

8) En el viaje de regreso tuvimos un fuerte temporal del N-NW durante toda la noche del 26 al 27 de julio, con intensos chubascos de lluvia y viento, mar gruesa del $\mathrm{N}$ y olas de 4 metros. Ello nos obligó a salir del rumbo y correrlo por la popa, para evitar los fuertes bandazos que producía en el barco. Tales circunstancias debieron provocar un nuevo desgaste físico en los pájaros que todavía teníamos a bordo.

El día 30 a las 09,55 h. (GMT), después de haber pasado el Estrecho de Gibraltar con un temporal de Levante de fuerza 8 de Beaufort (40 nu$\operatorname{dos}=74 \mathrm{~km} / \mathrm{h}$ ), y mar gruesa con olas de $6 \mathrm{me}-$ tros, decidimos soltar dos palomas a una distancia de 3 millas $(5,5 \mathrm{~km})$ del Cabo de Trafalgar y en demora verdadera $\mathrm{N}$. La tierra más próxima se encontraba a 0,5 millas $(0,9 \mathrm{~km})$, en la playa de El Palmar (entre Barbate y Conil). En el momento de la suelta había un viento de Levante de 16 nudos $(30 \mathrm{~km} / \mathrm{h})$.

La primera de las palomas, con número de anilla 5006563 se dirigió en vuelo muy bajo directamente a la costa, dando muestras de una gran fatiga. La segunda de ellas, con número de anilla 5006555 , 


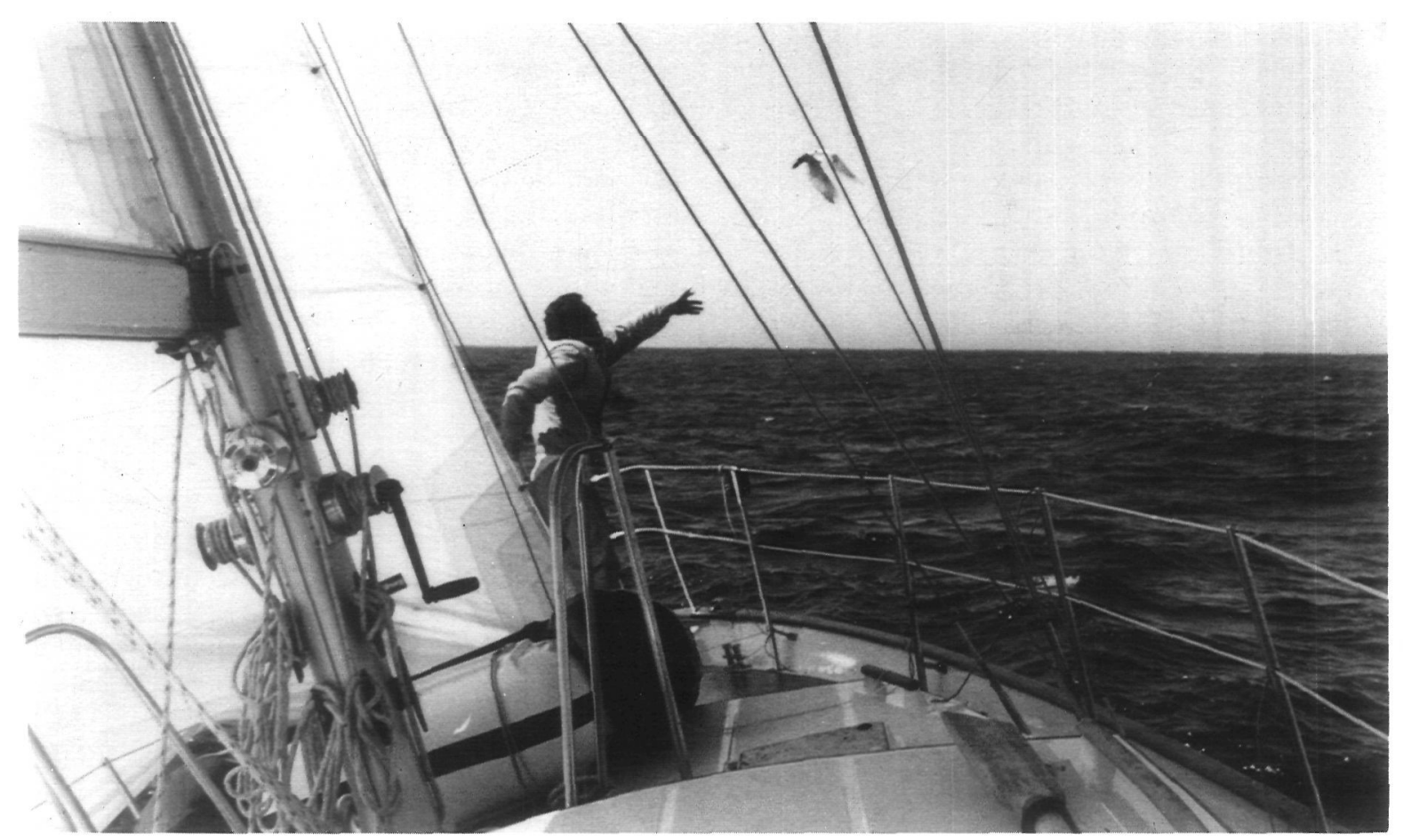

Fig. 9.-Suelta de palomas desde el barco Tartessos.

inició un corto vuelo, pero viró para regresar al bar$\mathrm{co}$, cayendo fatigada al agua, de donde la recogimos para enjaularla nuevamente (Fig. 5).

Con esta última experiencia quedaba claro que la utilidad de las aves tenía la limitación de las condiciones físicas en que se encontrasen y que, al cabo de muchos días en el mar, sometidas al fuerte desgaste de los temporales, podían perder eficacia como auxiliares del navegante.

De lo anteriormente descrito se deduce que para que el procedimiento de utilización de pájaros en la navegación sea eficaz, es necesario que cumplan unas ciertas condiciones. En primer lugar suponemos que debe ser determinante la correcta elección del pájaro idóneo, que en nuestro caso resultó la paloma común. En segundo lugar, es importante tener en cuenta que la seguridad depende en gran medida de ellos y que los navegantes antiguos deberían prestar una especial atención a su cuidado a bordo, y la alimentación. En tercer lugar, es importante que las aves estén en perfectas condiciones de vuelo, es decir, que hasta el momento de ser embarcadas hayan estado en libertad y quizás debidamente entrenadas. Finalmente, hay que tener en cuenta que en viajes largos, los pájaros pierden condiciones físi- cas y dejan de ser tan útiles orientadores como en las primeras jornadas. Ello plantearía el problema de cómo serían preparados para trayectos de múltiples singladuras o simplemente, si se les sustituiría por otros pájaros que estuviesen nuevamente en buenas condiciones físicas. En caso contrario este procedimiento de orientación sería sumamente limitado y utilizable tan sólo en la navegación local.

Existe la posibilidad, que nosotros no tuvimos en cuenta, de que las aves no estuviesen enjauladas, sino atadas por una pata o sujetas con un braguero, como se hace en la actualidad para emplearlas como reclamo en la caza de pájaros con red. De este modo estarían ejercitando las alas y podrían ser soltadas para buscar la costa sin las dificultades derivadas de transportarlas en jaulas. Si esto fue así, tendríamos un buen ejemplo gráfico en los numerosos modelos de barcos de la Cultura Nurágica en que las aves estản aparentemente sueltas y posadas en el mástil (Fig. 1). La misma imagen de la paloma u otra ave la encontramos en las Argonáuticas órficas, cuando Atenea envía un pájaro que se posa en el mástil y conduce con su vuelo a los compañeros de Jasón en uno de los momentos más difíciles por los que pasan (695 ss.) Los navegantes in- 
dios que hacían la ruta hacia Babilonia llevaban con ellos un cuervo en lo alto del mástil, para buscar la costa en caso necesario (disakaka-). En el BaveruJataka se cuenta: «Los nativos (de Babilonia) que los veían venir de vez en cuando y veían este pájaro (el cuervo) posado en lo alto del mástil, decían: tened cuidado con el color de ese pájaro» (KEEL, 1977, 81). Los pájaros que aparecen posados en los mástiles de los barcos egipcios en la época de Ramsés III son interpretados como auxiliares de los navegantes y no como elementos decorativos (KEEL, 1977, 1067.

De esta forma de llevar los pájaros a bordo puede haber surgido un tipo de competición que encontramos en el contexto de las navegaciones de la Edad del Bronce consistente en probar puntería, en los ratos de ocio, sobre una paloma que se tiene amarrada en la parte alta del mástil de la embarcación. Uno de los testimonios lo tenemos en la Ilíada, cuando Aquiles «clava en la arena de la playa un mástil de navío y amarra en su extremo una paloma. Luego, volviéndose a los que le rodeaban, les invitó a disparar sus flechas" (Ilíada, XXIII, 853) (33). La segunda mención se hace de forma más explícita por Virgilio y la paloma es atada al palo de la nave de Segesto, para servir de blanco mientras revolotea en el aire (VIRGILIO, Aen., V, 485) (34).

Tras la descripción de la experiencia anterior, en la que hemos hecho especial hincapié en las circunstancias que podrían tener utilidad a efectos de la orientación en el mar, conviene hacer algunas consideraciones sobre las palomas, que fueron las que aportaron el resultado más positivo de la experiencia. Para ello son de gran interés los datos aportados por los colombófilos y sus experiencias en la cría y suelta de palomas mensajeras (FERRÁN ANDREU, 1985).

El primer elemento importante para que la paloma esté en perfectas condiciones de vuelo hemos dicho que consiste en el buen estado del plumaje. En una paloma se distinguen tres clases de plumas: el plumón, las plumas de cobertura y las activas. Con las primeras aumentan su volumen en el curso del vuelo, reteniendo la mayor cantidad de aire en-

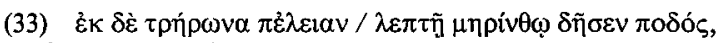

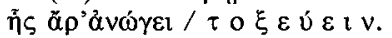

(34) Ingentique manu malum de naue Seresti / Erigit et uolucrem traiecto in fune columbam, / Quo tendant ferrum, malo suspendit $a b$ alto. tre ellas, con lo que se facilita lo que podríamos denominar su flotabilidad (FERRÁN ANDREU, 1985,15 ). Con las activas la paloma rema (plumas remeras de las alas), o dirige su vuelo (plumas timoneras de la cola). Así pues, unas buenas condiciones físicas pueden resultar insuficientes, si el plumaje no se encuentra en perfecto estado. En nuestra experiencia el relente y el salitre de los rociones mermaron considerablemente la calidad inicial de las plumas, a pesar del mucho cuidado que pusimos en evitarlo.

Otro factor de interés en el vuelo de aves como auxiliar de la navegación hemos dicho que serían sus condiciones físicas. En el caso de la paloma conviene subrayar que tiene millares de músculos, con los que mueve sus plumas, pero que solamente los pectorales constituyen la tercera parte de su peso total. $Y$ es con una buena musculatura, no muy voluminosa, pero bien ejercitada, con la que debe batir las alas ocho veces por segundo en su partida y seis veces por segundo en el curso del vuelo (FERRÁN ANDREU, 1985, 11). Resulta, obvio que el frío, los vaivenes y el excesivo tiempo en cautividad a bordo entumecen y debilitan las condiciones físicas iniciales. Por ello, cuando la seguridad del navegante dependía del estado de sus pájaros, es lógico que se les debería prestar una atención muy especial en este aspecto. Como hemos descrito, las últimas palomas soltadas por nosotros, después de quince días de viaje, ya no estaban en condiciones físicas ni con el plumaje adecuado para realizar vuelos a distancias largas.

La utilización de pájaros para buscar la costa desde alta mar se hace sirviéndose de su capacidad para elevarse a alturas en las que las brumas y nieblas bajas impiden la visión del litoral. Pero es desde allí donde con sus potentes ojos van a ver lo que los propios marineros serían incapaces de distinguir. Señalemos, por ejemplo, que la paloma es capaz de distinguir su palomar desde una distancia de $50 \mathrm{ki}$ lómetros (FERRÁN ANDREU, 1985, 32).

Por último, un dato a tener en cuenta a la hora de valorar la experiencia realizada por nosotros, es la de las distancias que vuela una paloma bien entrenada y la velocidad a que lo hace. En nuestro caso, la mayor distancia a costa, en la que la paloma indicó correctamente el rumbo más corto hacia tierra fue de 35 millas $(65 \mathrm{~km})$. Pero hay que decir que las distancias habituales para el vuelo de palomas mensajeras suelen ser muy superiores y alcanzan con frecuencia varios cientos de kilómetros. En cuanto 
a la velocidad, depende naturalmente de diversos factores, pero puede oscilar en torno a los $80 \mathrm{~km}$ a la hora (FERRÁN ANDREU, 1985, 119).

Si comparamos el vuelo de las palomas utilizadas por nosotros, con el de las aves descritas en los poemas más antiguos, en los que aparece el navegante perdido en alta mar y buscando tierra con la ayuda de unos pájaros, comprendemos que en tales relatos se nos describe situaciones reales, vividas por los marineros de hace tres y cuatro mil años. Efectivamente, la paloma que no encuentra tierra en la que posarse, revolotea en todas direcciones buscando de algún modo su libertad lejos del barco, pero regresa una y otra vez al punto de partida. Así lo describe el poema de Utnapishtim y de igual forma se comportan un cuervo y una paloma en el relato de Noé, o la paloma en el mito de Deucalión. En todos ellos la historia concluye cuando el pájaro que han soltado ya no regresa al barco. A partir de ese momento ya pueden dar gracias al cielo por su salvación; ya conocen el rumbo que deben seguir para encontrar tierra. Es la dirección que, como a Eneas, le marcan unas palomas que se pierden en el horizonte.

Quedaría por explicar cómo se le ha ocurrido al hombre la utilización de pájaros en el mar, y dónde podríamos situar el origen de esta práctica. Para ello quizá sea un buen indicio saber que en la Antigüedad la suelta de pájaros se hacía igualmente en tierra, pero con la finalidad de buscar agua en el desierto. Por este procedimiento se orientó Alejandro hacia el oráculo de Ammón en el oasis de Siwa (ESTRABÓN, XVII, 1, 43) y del mismo modo encuentra Salomón a la Reina de Saba en las montañas del Yemen, cuando estaba soltando pájaros para buscar agua mientras cruzaba el desierto de Arabia (HORNELL, 1946, 148). Lo más lógico es, por consiguiente, que los navegantes utilizasen en alta mar desde el tercer milenio a. de C. una antiquísima técnica conocida con anterioridad y utilizada en tierra firme.

Como posible recuerdo de la primitiva utilización de los pájaros para la localización del agua, cabe recordar el episodio del castigo que infringe Apolo al cuervo, que, al haber sido enviado a por agua, se detiene en un campo de grano a esperar que madure y no regresa (PAUSANIAS, II, 26, 6; CLAUDIO ELIANO, I, 47). El dios desde entonces le castiga a pasar sed durante el verano.

El envío de un cuervo que no regresa, nos invita a relacionar de algún modo las historias y leyendas del Mediterráneo con otra abundante serie de narraciones similares en la que se cuenta la gran catástrofe de un diluvio o una inundación en las diversas culturas de la América precolombina. Frazer las reúne $\mathrm{y}$ analiza de manera exhaustiva (FRAZER, 1981, 128 ss.) pero atribuye la presencia de ciertas semejanzas formales a la influencia de misioneros cristianos de los que no tenemos noticia. Es cierto que historias de inundaciones y diluvios con consecuencias catastróficas son abundantes y prácticamente universales debido a que con ello se mantienen recuerdos vividos realmente por algún grupo humano o porque así se explica la presencia de fósiles marinos en lugares muy alejados de la costa. Pero no se suele considerar la posibilidad de que el empleo de aves por parte de los que se salvan en una balsa pueda responder a un procedimiento elemental de navegación, y por ello se atribuye a influencias del relato bíblico a través de las misiones. Entre las narraciones recogidas por Frazer hay una de los indios cora en la que tras el diluvio es enviado un cuervo, que no regresa porque se queda devorando los cadáveres (FRAZER, 1981, 144). También los indios mandan cuentan la historia de una inundación en el que el único superviviente se salva en una canoa y suelta una tórtola o una paloma torcaz (FRAZER, 1981, 148). Los montaguais del Canadá tienen un héroe que sobrevive a una inundación y que suelta un cuervo (FRAZER, 1981, 151). Los indios salteaux o chippewa cuentan la historia del hechicero Wis quien emplea en el mismo trance un somormujo atado a la pata por un cordel y luego un cuervo (FRAZER, 1981, 155). Los indios ojibwas, que habitaban al oeste del lago Ontario narraban la historia de Nenebojo, que se salvó en una balsa con una pareja de animales de cada especie y envió al final de la catástrofe un cuervo, que no regresó y un halcón. Finalmente, entre los indios dené se salva un anciano que suelta un cuervo desde su canoa, que no regresa porque se queda saciando el hambre en los cadáveres flotantes (FRAZER, 1981, 157).

Con los anteriores ejemplos no pretendemos hacer viajar universalmente la historia de un diluvio, sino apuntar la posibilidad de que la suelta de ciertas aves al final de la narración responda a un procedimiento elemental de navegación, que puede haberse extendido por diversas vías, antes que pensar en contaminaciones bíblicas debidas a los misioneros, como parece ser la idea más difundida. 
Entre los datos recogidos por nosotros acerca de animales empleados en el barco, mencionábamos más arriba la presencia de unos monos en diversos lugares y culturas de la Antigüedad. Quizá la alusión que se hace a monos y pavos (?) en el Libro I de los Reyes (I, 10, 22) tenga más sentido en el contexto de su posible utilidad, que en el de un comercio exótico e inútil. Por esta razón embarcamos en el Tartessos una mona del tipo cercopitecus aethiops, que nos fue cedida por el Ayuntamiento de Cádiz, a fin de analizar su comportamiento durante el viaje (35).

En los exvotos sardos se ve con claridad que los monos están haciendo gestos inconfundibles de observar el horizonte (RINGEL, 1986, n. ${ }^{\circ}$ 54), pero no se les ve participar en labores auxiliares relacionadas con el gobierno del barco, como han sugerido algunos autores al referirse a los monos que utilizaban los egipcios en la flota del Punt (BRUNNER-TRAUT, 1975). $Y$ es precisamente la gran capacidad de observación y la atenta vigilancia en que están continuamente los monos de esta especie (SCHULTZ, 1979, 291) (36), la que a nuestro juicio es utilizada por el marinero de la Antigüedad.

Nosotros habíamos pensado en un principio, que los monos podían haber sido empleados para divisar el horizonte y descubrir tierra con su aguda vista o con su especial sensibilidad. Pero la experiencia de llevar esta macaca durante catorce días en una embarcación de proporciones muy reducidas, nos sirvió para analizar detenidamente su comportamiento. Esto fue posible principalmente en el viaje de regreso, en que la tuvimos completamente suelta, y comprobábamos así la conducta de un animal que no se encontraba en su medio y que estaba en todo momento pendiente de la menor anomalía en su entorno, ya se tratase de algo dentro del barco o fuera de él. Por ello pensamos que los monos, si de algo podían servir al navegante antiguo, es para tener en ellos unos vigilantes incansables y muy eficaces. Nuestra experiencia no aportó datos concretos que no hayan sido estudiados por quienes han tratado en otras ocasiones acerca de la conducta de los primates.

(35) Agradecemos a D. José Lado su cooperación en todo momento para la realización de este proyecto.

(36) VIRÉ, 1980, aporta datos tomados de al-Damiri, Hayat, 243-4, sobre la domesticación de monos del tipo cercopitecus aethiops, traidos desde las costas occidentales africanas al sur del Yemen y Omán, para utilizarlos en tareas tales como mover la piedra de los molinos o vigilar los comercios.

\section{BIBLIOGRAFÍA}

ALVAR EZQUERRA, J. 1981: La navegación prerromana en la Peninsula Ibérica: colonizadores e indigenas. Madrid.

ANDRÉ, J. y FILLIOZAT, J. 1980: Pline l'Ancien, Hist Nat. París.

ANTI, C. 1920: «Athena Marina e Alata», Rendiconti Acc. dei Lincei, 270-318.

ARNAL, J. 1976: Les Statues-menhirs, Hommes et Dieux, Toulouse.

AUBET, M. ${ }^{a}$ E. 1984: «Los hallazgos micénicos en Italia: Estado actual de la cuestión», BSAA. 157-162.

BARNETT, R. D. 1958: «Early Shipping in the Near East», $A n-$ tiquity, 32, 220-230.

BARTRA, A. 1972: La Epopeya de Gilgamesh, Barcelona.

BIANCHI BANDINELLI, R. y GIULIANO, A. 1974: Los Etruscos y la Italia anterior a Roma, Madrid, ed. esp.

BIANCO PERONI, V. 1970: Die Schwerter in Italien. Le spade nell'Italia Continentale, Munich.

BISI, A. M. 1978: «Elements anatoliens dans les bronzes nouragiques de Sardaigne», Proceedings of the $X$ International Congress of Classical Archaeology, I, Ankara-Izmir.

BITTEL, K. 1976: Los Hititas, Madrid, ed. esp.

BLOCH, R. 1973: Los Etruscos, ed. esp. Barcelona.

BOARDMAN, J. 1985: Die Keramik der Antike, Freiburg.

BOUZEK, J. 1985: The Aegean, Anatolia and Europe: Cultural Interrelations in the Second Millenium B. C., Göteborgpraga.

BRUNNER-TRAUT, E. 1975: «Affe», Lexikon der Ägyptologie, Wiesbaden.

CARLMEYER, P. 975: «Gilgames», Reallexikon der Assyriologie, Berlín.

CASON, L. 1973: Ships and Seamenship in the Ancient World, Princeton.

CHERNISS, H. y HEMBOLD, C. 1984: Plutarc's Moralia, Londres, 3. ${ }^{\mathrm{a}}$ ed.

COLONNA, G. 1981: «Quali Etruschi a Roma, Apendice II; La Barchetta nuragica di Porto ritrovata», Gli etruschi e Roma. Atti del'incontro di studio in onore di Massimo Pallotino, Roma.

D'ARCY-THOMSON, W.: 1936, A Glossary of Greek Birds Oxford.

DETIENNE, M. 1970: «Le Navire d'Athéna», Rev. Hist. Rel. 133-177.

FERNÁNDEZ MIRANDA, M. 1978: Secuencia Cultural de la Prehistoria de Mallorca, Madrid.

FERRÁN ANDREU, J. M.: 1985, Las palomas mensajeras, Barcelona.

FERRI, S. 1965: «Problemi generali intorno a la genesi della civiltà nuragica», Studi classici e Orientali, 14, 261 ss.

FILLIOZAT, J. 1949: «Les Echanges de l'Inde et l'Empire Romain aux premiers siècles de l'ere Chrêtienne, Revue Historique, $73,1-29$.

FRANKFORT, H. 1939: Cylinder Seals, Londres.

FRAZER, J. G. 1981: El Folklore en el Antiguo Testamento, Madrid-Méjico, ed. esp.

FREEDMAN, R. D. 1973: «The Dispatch Reconnaisance Birds in Gilgamesh XI», The Journal of the Ancient Near Eastern Society of Columbia University, 39, 123-129.

GARCÍA Y BELLIDO, A. 1948: Hispania Graeca, Barcelona. GEORGIEV, V. I. 1962: Hethitisch und Etruskisch, Sofia.

GÖTTLICHER, A. 1978: Materialien für ein Corpus der Schiffsmodelle im Altertum, Mainz.

GONZÁLEZ PRATS, A. 1985: «Los nuevos asentamientos del final de la Edad del Bronce: problemática cultural y crono- 
lógica), Arqueología del País Valenciano: Panorama y perspectivas, Alicante.

GRAY, D. 1974: Seewessen, Archeologia Homerica, Göttingen.

GROSJEAN, R. 1962: «Les armes portées par les estatuesmenhirs de Corse», Rev. Arch. 2, 1 ss.

GROSJEAN, R. 1966-a: La Corse avant l'Histoire, París.

GROSJEAN, R. 1966-b: «Recent Work in Corsica» Antiquity, $40,190$.

HEURGON, J. 1981: «Introduction a la methode de Massimo Pallotino». Gli Etruschi e Roma. Incontro di studio in onore di Massimo Pallotino, Roma.

HOLLOWAY, R. 1981: Italy and the Aegean, Lovaina.

HORNELL, J. 1946: «The Role of Birds in Early Navigation», Antiquity, 20, 142-149.

HUTCHINSON, R. W. 1962: La Creta Prehistórica, Londres, ed. esp. Méjico 1978.

IENTILE, M. G. 1986: La Pirateria Tirrenica. Momenti e Fortuna, Suplemento a Kokalos, 6, Roma.

JONES, G. 1985: A History of the Vikings, Oxford, $2 .^{\mathrm{a}} \mathrm{ed}$.

KEEL, O. 1977: Vögel als Boten, Göttigen.

KRAPPE, A. H. 1936: «Les dieux au courbeau chez les Celtes», Rev. Hist. Rel. 94.

LEVIN, D. N. 1971: Apolonio's Argonautica re-examined, Leiden.

LILLIU, G. 1962: «Las Nuragas», Ampurias, 24, 67 ss.

LILLIU, G. 1966: Scultura della Sardegna Nuragica, Verona.

LILlIU, G. 1980: La Civiltà dei Sardi, Turin, 2. ${ }^{\mathrm{a}}$ ed.

LUYSTER, R. 1965: «Symbolic Elements in the Cult of Athena», History of Religions, 133-163.

MARMS, G. J. 1953: «The Navegation of the Norsemen», $M a-$ riners' Mirror, 38, 128 ss.

Mc CRINDLE, J. M. 1901: Ancient India as described in Classical Literature, Westminster.

MÜLLER-KARPE, H. 1982: «Zur Seefahrt im 3. und 2. Jahrtausend vor Chr.» Zur Geschichtlichen Bedeutung der frühen Seefahrt, Munich.

NELSON, H. 1942: «The Naval Battle Pictured at Medinet Habu» Journal of Near Eastern Studies, 2, 40 ss.

ORMEROD, H. A. 1978: Piracy in the Ancient World, Liverpool.

ORTHMANN, W. 1976-1980: «Kanish, karum», Reallexikon der Assyriologie, Berlín.
PALLOTTINO, M. 1968: Etruscologia, Milán, 6. ${ }^{\circledR}$ ed.

PEMAN, C. 1931: «Sobre la antigüedad de la fundación de Cádiz», $B R A H, 98$.

PEMAN, C. 1954: «Las fuentes literarias de la antigüedad y la fundación de Cádiz», Comisión de Estudos e Investigaciones de la Historia de Cádiz, Madrid.

PERUZZI, E. 1980: Miceneans in Early Latium, Roma.

PICARD, C. 1982: «Les navigations de Carthage vers l'Ouest. Carthage et le Pays de Tharsis aux VIII-VI siècles», Phöniker im Westen, Maguncia, 167 ss.

RINGEL, J. 1986: Ancient Sardinian Art 3000-300 B. C. E. The Elie Borowski Collection, Haifa.

RODRÍGUEZ ADRADOS, F. 1987: «Ibico 61 y el influjo del Gilgamesh en Grecia». Aula Orientalis, 5-1, 5 ss.

RUIZ DE ELVIRA, A. 1982: Mitología Clásica, Madrid.

SANDARS, N. K. 1978: The Sea Peoples, Londres.

SCHÜLE, W. 1968: Navegación primitiva y visibilidad de la tierra en el Mediterráneo», XI Congreso Nacional de Arqueología, Mérida, 1968, Zaragoza, 1970, 449-462.

SCHULTZ, A. H. 1979: Los Primates, Barcelona.

SÖLVER, C. V. y MARCUS, G. J. 1958: «Dead Reckoning and the Ocean Voyages in the Past», Mariner's Mirror, 44, 32 ss.

TORELLI, M. 1970: «Gravisca», «Nouvi tesori dell'antica Tuscia), Viterbo.

TORELLI, M, 1984: Storia degli Etruschi, Roma-Bari.

TÜMPEL, 1905: «Deukalion», RE, 5, 272.

VAGNETTI, L. 1980: «Micenean imports in central Italy», en F. PERUZZI, Miceneans in Early Latium, Roma.

VAGNETTI, L. 1982: «Quindici anni di studi e ricerche sulle relazioni tra il mondo egeo e l'Italia protoistorica», Magna Grecia e Mondo Miceneo, Taranto.

VIRÉ, F. 1980: «Kird», Encyclopedie de l'Islam, 5, Paris.

VERLINDEN, C. 1985: «The Indian Ocean. I The Ancient Period and the Middle Ages», XVI Congrés International des Sciencies Historiques, Rapports, I, Stuttgart.

WACHSMANN, S. 1981: "The Ships of the Sea Peoples», International Journal of Nautical Archaeology and Underwater Exploration, 10-3, 187-220.

WACHSMANN, S. 1982: "The Ships of the Sea Peoples: additional notes», International Journal of Nautical Archaeology and Underwater Exploration, 11-4, 297-304. 